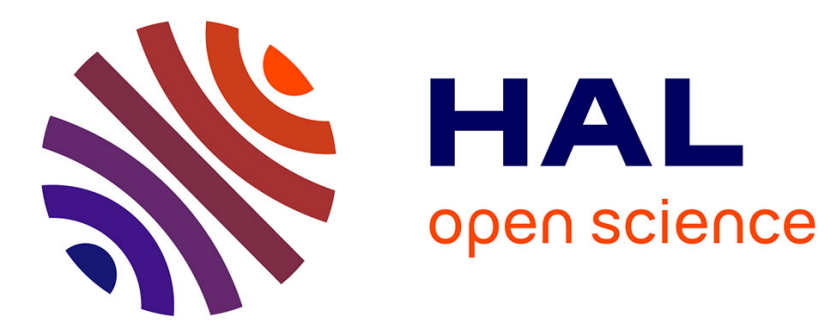

\title{
Complete Active Space Wavefunction-Based Analysis of Magnetization and Electronic Structure
}

Frédéric Gendron, Hélène Bolvin, Jochen Autschbach

\section{To cite this version:}

Frédéric Gendron, Hélène Bolvin, Jochen Autschbach. Complete Active Space Wavefunction-Based Analysis of Magnetization and Electronic Structure. Organometallic Magnets, 64, pp.355-390, 2019, Topics in Organometallic Chemistry, 978-3-030-26008-8. 10.1007/3418_2018_6 . hal-02390719

\section{HAL Id: hal-02390719 https://hal.science/hal-02390719}

Submitted on 18 Feb 2020

HAL is a multi-disciplinary open access archive for the deposit and dissemination of scientific research documents, whether they are published or not. The documents may come from teaching and research institutions in France or abroad, or from public or private research centers.
L'archive ouverte pluridisciplinaire HAL, est destinée au dépôt et à la diffusion de documents scientifiques de niveau recherche, publiés ou non, émanant des établissements d'enseignement et de recherche français ou étrangers, des laboratoires publics ou privés. 


\title{
Chapter 0 \\ Complete Active Space (CAS) Wavefunction-Based Analysis of Magnetization and Electronic Structure
}

Frédéric Gendron, Hélène Bolvin, and Jochen Autschbach

\begin{abstract}
A theoretical framework for the generation of natural orbitals, natural spin orbitals, as well as orbital- and spin-magnetizations from multi-configurational ab-initio wavefunction calculations including spin-orbit coupling is presented. It is shown how these computational orbital and magnetization tools can be used to interpret and rationalize the magnetic properties of selected complexes containing transition metals, lanthanides, and actinides.
\end{abstract}

\section{Units, Notation, Acronyms}

The reader is assumed to be familiar with basic concepts of quantum mechanics and computational chemistry. SI or Hartree atomic units (au) are employed. Nuclear motion is ignored in the discussion because our focus is on the electronic structure and bonding, and the resulting magnetic properties. The symbols $\cdot$ and $\times$ indicate inner and outer products, respectively, for vectors and matrices or tensors. Bold-italic notation such as $\boldsymbol{r}, \hat{\boldsymbol{S}}, \boldsymbol{\mu}$ is used for vectors and vector quantum operators. Uprightbold such as $\mathbf{a}, \mathbf{G}, \boldsymbol{\mu}$ is used for matrices and rank-2 tensors. The EPR pseudo-spin operator is denoted as $\mathbb{S}$.

The following acronyms are used in the text:

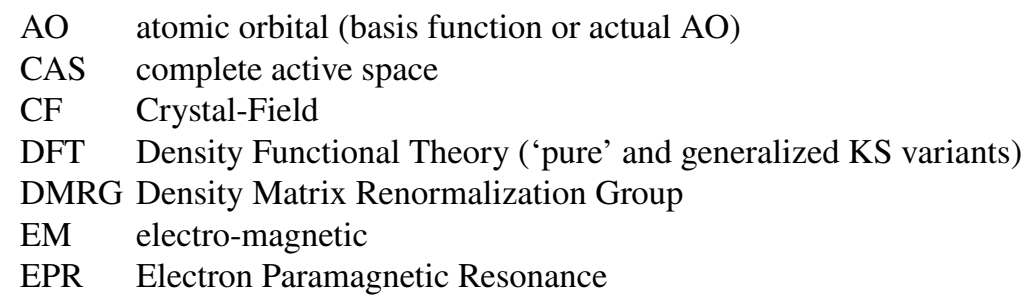

Please address correspondence to Jochen Autschbach, Department of Chemistry, University at Buffalo, State University of New York, e-mail: jochena@buffalo. edu 
ES Excited State

GIAO gauge-including atomic orbital

GS Ground State

HF Hartree-Fock

HFC hyperfine coupling

HOMO Highest Occupied Molecular Orbital

KD Kramers Doublet

KS Kohn-Sham

LR Linear Response

MO molecular orbital

NMR Nuclear Magnetic Resonance

NR non-relativistic (calculation excluding any relativistic effects)

PV principal value (of a tensor)

PAS principal axis system (of a tensor)

QM quantum mechanical (e.g. in reference to Dirac, Schrödinger Eqs.)

SO spin-orbit (usually means calculation also includes SR effects)

SOS sum over states

SR scalar relativistic (relativistic calculation without $\mathrm{SO}$ effects)

TIP Temperature-Independent Paramagnetism

WFT wave-function theory

ZFS zero-field splitting

\subsection{Introduction}

Metal complexes with interesting magnetic properties tend to have complicated electronic structures that require a multi-configurational (multi-reference) wavefunction description including scalar relativistic (SR) effects and spin-orbit (SO) coupling (also a relativistic effect). In the context of this article, the SO level of theory is meant to include both SR and SO effects. The complete active space (CAS) wavefunction theory (WFT) framework, whereby one performs a full or restricted configuration interaction within a chosen active space of orbitals, or on CAS-like approach utilizing a DMRG framework, [1-3] is the method of choice for such magnetic property calculations. This is especially true for orbitally degenerate electronic states, which are generally not well described by single reference methods such as Kohn-Sham density functional theory (KS-DFT) or standard coupled-cluster methods. [4] However, SO multi-reference wavefunctions are complex, in the usual meaning of complicated or intricate, as well as in the mathematical sense. Unlike KS-DFT calculations however, multi-reference SO wavefunctions have traditionally not been amenable to an intuitive chemically inspired analysis in terms of molecular orbitals.

In recent years, analysis tools in terms of orbitals that are familiar to chemists have become available to dissect the electron density, as well as spin-densities and magnetization densities that arise from the orbital angular momentum, based on sophisticated SO multi-reference wavefunction calculations. Furthermore, the magne- 
tization densities arising from the spin and orbital angular momentum in SO CAS calculations can be visualized. In combination with the orbitals, detailed information can be extracted from ab-initio calculations about how calculated magnetic susceptibilities and electronic magnetic moments ( $g$-factors) are related to the electronic structure, and ultimately to the structure of, and chemical bonding within, a metal complex.

In Section 0.2, some theoretical aspects underlying these analyses are presented. Section 0.3 presents a variety of case studies spanning the range from $3 \mathrm{~d}$ metals to actinides.

\subsection{Theoretical Methods}

We assume that the metal complex is oriented within the laboratory coordinate frame such that its principal magnetic axes coincide with the $x, y$, and $z$ direction. This can be achieved, for instance, by first calculating the magnetic susceptibility tensor in its Cartesian $3 \times 3$ matrix representation (components $x x, x y=y x, x z=z x, y y, y z=$ $z y, z z$ ). Diagonalization of the tensor gives its principal values (the eigenvalues) and the principal axis system (PAS, the eigenvectors). The complex is then rotated such that the PAS coincides with the unit vectors of the Cartesian laboratory coordinate frame. Furthermore, the components of degenerate states can be chosen to diagonalize the Zeeman operator for the magnetic field along a selected magnetic axis, as described, for instance, in Reference 5.

Static homogeneous external magnetic fields can usually be described well by the corresponding nonrelativistic orbital and spin Zeeman operators, even if the electronic structure calculation of the complex requires a treatment of relativistic effects. The magnetic moment operator that describes the interaction of the electrons with a magnetic field in direction $u \in\{x, y, z\}$ is in the 'nonrelativistic with spin' formalism given by

$$
\begin{aligned}
\hat{\mu}_{u} & =-\mu_{B} \hat{M}_{u}=-\mu_{B}\left(\hat{L}_{u}+g_{e} \hat{S}_{u}\right) \\
\hat{M}_{u} & =\hat{L}_{u}+g_{e} \hat{S}_{u}
\end{aligned}
$$

Here, $\mu_{B}=e \hbar /\left(2 m_{e}\right)$ is the Bohr magneton, and $g_{e}=2.00231930436153(53)$ is the free electron $g$-value [6]. The small deviations of the free electron $g$-value from two are due to quantum electrodynamics (QED) corrections to the Dirac relativistic treatment. The operators $\hat{L}_{u}$ and $\hat{S}_{u}$ are the dimensionless one-electron operators for the $u$-component of the orbital angular momentum and the spin angular momentum, respectively.

Let $\psi$ be an electronic ground or excited state wavefunction of the complex, or a component of the respective state if it is degenerate. Based on Equation (0.1), orbital and spin magnetizations can be defined as follows: 


$$
\begin{aligned}
& m_{u}^{L}(\boldsymbol{r})=\int \psi^{*} \hat{L}_{u} \psi d \tau^{\prime} \\
& m_{u}^{S}(\boldsymbol{r})=g_{e} \int \psi^{*} \hat{S}_{u} \psi d \tau^{\prime}
\end{aligned}
$$

The notation $d \tau^{\prime}$ indicates an integration over all electron spin degrees of freedom, and all but one electron position degrees of freedom. The resulting functions therefore depend on a single electron coordinate. The procedure for calculating the magnetizations is similar to how the electron density $\rho$ is defined in terms of the wavefunction:

$$
\rho(\boldsymbol{r})=N \int \psi^{*} \psi d \tau^{\prime}
$$

In the equation for the electron density, $N$ is the number of electrons of the system.

The three functions, $m_{u}^{L}(\boldsymbol{r}), m_{u}^{S}(\boldsymbol{r})$, and $\rho(\boldsymbol{r})$, are real functions of 3D space and therefore they can be visualized easily, for example in cut-planes or as 3D isosurfaces. The volume integrals of these functions over 3D space give

$$
\begin{gathered}
\int m_{u}^{L}(\boldsymbol{r}) d V=\left\langle L_{u}\right\rangle \\
\int m_{u}^{S}(\boldsymbol{r}) d V=g_{e}\left\langle S_{u}\right\rangle \\
\int \rho(\boldsymbol{r}) d V=N
\end{gathered}
$$

The integral over the electron density gives the electron number. In the other two cases, the integration gives the expectation value of a component of the orbital or spin angular momentum. We write $g_{e}$ in the equations in this section, but in the calculations of the individual magnetization densities the QED corrections are neglected and $g_{e}=2$ is used for the spin magnetization. Per Equation (0.1), the magnetic moment expectation value is obtained from the magnetizations via

$$
\left\langle\mu_{u}\right\rangle=-\mu_{B}\left\langle M_{u}\right\rangle \text { with }\left\langle M_{u}\right\rangle=\int\left[m_{u}^{L}(\boldsymbol{r})+m_{u}^{S}(\boldsymbol{r})\right] d V
$$

If $\psi$ is a component of a degenerate state of interest in a computational study of a paramagnetic metal complex, then arbitrary unitary transformations among the state components can be applied without loss of generality. However, the functions $m_{u}^{L}(\boldsymbol{r})$ and $m_{u}^{S}(\boldsymbol{r})$ may not be invariant under such transformations. The electron density $\rho(\boldsymbol{r})$ for the components of orbitally degenerate states may also be subject to symmetry breaking if the full point group of symmetry of the complex is not enforced in the calculations. Many quantum chemistry programs do not support non-abelian symmetry groups. For example, for a non-bonding orbital of a $d^{1}$ transition metal or an $f^{1} f$-element configuration with linear metal coordination and a principal rotational symmetry axis of order $>2 \ell$, with $\ell=2$ or 3 , respectively, $m_{\ell}$ is a good 
quantum number at the SR level. Accordingly, the calculated electronic structure should reflect the rotational symmetry. The $d$ and $f$ orbitals can then be classified by their symmetry $\sigma, \pi, \delta,-$ and $\phi$, for $f$ orbitals - with respect to the rotational axis, corresponding to $\left|m_{\ell}\right|=0,1,2,3$. (This notation is often convenient even when the actual symmetry is lower.) The $\pi, \delta$, and $\phi$ levels are doubly degenerate. Single (or triple) occupations of these levels correspond to an orbitally degenerate state. In order for each state component to have a rotationally symmetric electron density individually, the degenerate orbitals must be evenly occupied (e.g. 0.5 each for a $d^{1}$ or $f^{1}$ configuration) if they are the usual real $d$ or $f$ orbitals. However, NR and SR CAS calculations for such a non-bonding degenerate $d^{1}$ or $f^{1}$ example will likely produce two state components in which one of the real $d$ or $f$ orbitals is occupied and the counterpart of the same symmetry is unoccupied. As a consequence, the rotational symmetry is broken in the individual state components. Well-defined spin and orbital magnetization densities can be generated easily after a subsequent treatment of the SO interaction, which generally requires complex wavefunction coefficients anyway. The components of the degenerate state are then chosen to diagonalize the magnetic moment operator matrix for a given field direction. In the case studies of Section 0.3, we diagonalize the $u$ component of the magnetic moment operator prior to generating $m_{u}^{L}(\boldsymbol{r})$ and $m_{u}^{S}(\boldsymbol{r})$. Unless stated otherwise, the resulting state component with the most positive $\left\langle S_{u}\right\rangle$ is then chosen for further analysis. For axial complexes, the procedure tends to generate linear combinations of the SR states such that, for the $d^{1}$ or $f^{1}$ example, equal occupations of $\pi, \delta$, or $\phi$ metal orbitals are achieved, which corresponds to forming the angular momentum eigenfunctions from the real $d$ or $f$ metal orbitals.

When NR or SR calculations generate spin eigenfunctions with well-defined quantum numbers $S$ and $M_{S}$, then $\left\langle S_{u}\right\rangle=M_{S}$ if $u$ is the chosen projection quantization axis. In quantum chemistry, $u=z$ is the default choice. The function $m_{z}^{S}$ is then nothing but the familiar spin density. When the magnetic moment operator for a degenerate state is diagonalized, the direction of the magnetic field defines the quantization axis for the spin and the orbital angular momentum. In relativistic calculations where the effects from spin-orbit (SO) coupling are included in the wavefunctions, spin and orbital angular momentum cease to be good quantum numbers. Of course, the corresponding expectation values can still be calculated, and they are meaningful because of their contributions to the magnetic moment.

When an atomic orbital (AO) basis set $\left\{\chi_{\mu}\right\}$ with real functions is used, as it is customary in quantum chemistry, the electron density and the spin magnetization can be expressed in the $\mathrm{AO}$ basis in terms of the elements of density matrices:

$$
\begin{aligned}
\rho & =\sum_{\mu \nu} \chi_{\mu} \chi_{\nu} D_{\nu \mu}^{\rho} \\
m_{u}^{S} & =\sum_{\mu \nu} \chi_{\mu} \chi_{\nu} D_{u, \nu \mu}^{S}
\end{aligned}
$$

It is important to note that the action of the spin operator is fully considered in Equation $(0.2 b)$, and that subsequently all spin degrees of freedom are integrated over. 
Therefore, there are no spin degrees of freedom explicitly contained in $m_{u}^{S}$ anymore, but of course the function implicitly depends on the action of the spin operator because of Equation (0.2b). The matrices $\mathbf{D}^{\rho}$ and $\mathbf{D}_{u}^{S}$ which are formed by the elements $D_{\nu \mu}^{\rho}$ and $D_{u, \nu \mu}^{S}$, respectively, are real and symmetric, and therefore they can be diagonalized in order to reduce the double sums in Equations $(0.7 \mathrm{a}, 0.7 \mathrm{~b})$ to single sums:

$$
\begin{aligned}
& \rho(\boldsymbol{r})=\sum_{p} n_{p}\left[\varphi_{p}(\boldsymbol{r})\right]^{2} \quad \text { with } \sum_{p} n_{p}=N \quad \text { and } \int\left[\varphi_{p}(\boldsymbol{r})\right]^{2} d V=1 \\
& m_{u}^{S}(\boldsymbol{r})=\sum_{p} n_{u, p}^{S}\left[\varphi_{u, p}^{S}(\boldsymbol{r})\right]^{2} \text { with } \sum_{p} n_{u, p}^{S}=g_{e}\left\langle S_{u}\right\rangle \text { and } \int\left[\varphi_{u, p}^{S}(\boldsymbol{r})\right]^{2} d V=1
\end{aligned}
$$

The orbitals (one-electron functions) $\varphi_{p}$ are the natural orbitals (NOs), and the numbers $n_{p}$ are the corresponding populations (occupations). We refer to the eigenfunctions $\varphi_{u, p}^{S}$ of the spin magnetization components as natural spin orbitals (NSOs). The numbers $n_{u, p}^{S}$ are the corresponding spin populations. The orbitals are chosen to be normalized, such that the populations of the NOs add up to the total number of electrons, and the sum of the spin populations gives $g_{e} \simeq 2$ times the expectation value for spin component $u$. Furthermore, the orbitals are chosen to be real such that for metal $d$ and $f$ orbitals they resemble the orbitals familiar from textbooks. We remind the reader that the NO populations are not necessarily integers, because the wavefunctions may be composed of several, or even very many, Slater determinants, due to a combination of static and dynamic correlation, and SO coupling. The spin populations are likewise not necessarily integers. Examples for how the NO and NSO populations can help with the analysis of complicated electronic structures are provided in Section 0.3.

The orbital magnetization requires additional consideration, because of the derivative term in the (dimensionless) one-electron orbital angular momentum component operator

$$
\hat{L}_{u}=-i \sum_{k=1}^{N}\left[\boldsymbol{r}_{k} \times \nabla_{k}\right]_{u}
$$

The notation indicates that the component $u$ of the vector $\boldsymbol{r}_{k} \times \boldsymbol{\nabla}_{k}$ is used to construct $\hat{L}_{u}$. The operator $\hat{L}_{u}$ is imaginary, self-adjoint for square integrable functions, and spin-independent. It is possible to construct, from a many-electron wavefunction, a one-particle density matrix $\mathbf{D}^{J}$ that can be used to calculate the expectation value of an imaginary operator such as $\hat{L}_{u}$ as follows:

$$
\left\langle L_{u}\right\rangle=-i \sum_{\mu \nu}\left\langle\chi_{\mu}\left|[\boldsymbol{r} \times \nabla]_{u}\right| \chi_{\nu}\right\rangle D_{v \mu}^{J}
$$

The matrix formed by the integrals $\left\langle\chi_{\mu}\left|[\boldsymbol{r} \times \nabla]_{u}\right| \chi_{\nu}\right\rangle$ over the real AO basis is real and antisymmetric, i.e. upon multiplication with the factor $-i$ one obtains a purely imaginary Hermitean matrix. Likewise the matrix $\mathbf{D}^{L}$ is imaginary and antisymmetric, such that the expectation value is real. We use a superscript $J$ for this matrix in 
order to associate it with the paramagnetic current density defined below, as it is not specific to the orbital angular momentum. The matrix $\mathbf{D}^{J}$ with elements $D_{\nu \mu}^{J}$ can be used to calculate expectation values of any spin-independent quantum operator that is imaginary and has an antisymmetric matrix representation in the AO basis. Since the matrix $\mathbf{D}^{J}$ is Hermitian, it can be diagonalized to give a set of complex eigenfunctions $\varphi_{p}^{J}$, and real eigenvalues $n_{p}^{J}$, such that the double sum of Equation 0.11 reduces to:

$$
\begin{aligned}
\left\langle L_{u}\right\rangle & =-i \sum_{p} n_{p}^{J} \int \varphi_{p}^{J *}[\boldsymbol{r} \times \boldsymbol{\nabla}]_{u} \varphi_{p}^{J} d V \\
& =-\frac{i}{2} \sum_{p} n_{p}^{J} \int\left(\varphi_{p}^{J *}[\boldsymbol{r} \times \boldsymbol{\nabla}]_{u} \varphi_{p}^{J}-\varphi_{p}^{J}[\boldsymbol{r} \times \boldsymbol{\nabla}]_{u} \varphi_{p}^{J *}\right) d V
\end{aligned}
$$

The antisymmetrized form in the second line of Equation (0.12) is more commonly used when the orbital magnetization is re-cast in terms of the real-valued paramagnetic current density. The latter is defined in the context of the present discussion as the real-valued vector field

$$
\boldsymbol{j}(\boldsymbol{r})=-\frac{i}{2} \sum_{p} n_{p}^{J}\left(\varphi_{p}^{J *} \nabla \varphi_{p}^{J}-\varphi_{p}^{J} \nabla \varphi_{p}^{J *}\right)
$$

In terms of the paramagnetic current density, the orbital magnetization is given as

$$
m_{u}^{L}=[\boldsymbol{r} \times \boldsymbol{j}]_{u}=\sum_{p} n_{p}^{J}\left[\boldsymbol{r} \times \boldsymbol{j}_{p}\right]_{u}
$$

A per-orbital paramagnetic current density can be defined accordingly as

$$
\boldsymbol{j}_{p}(\boldsymbol{r})=-\frac{i}{2}\left(\varphi_{p}^{J *} \nabla \varphi_{p}^{J}-\varphi_{p}^{J} \nabla \varphi_{p}^{J *}\right)
$$

The numbers $n_{p}^{J}$ do not have as specific of a definition as the NO and NSO populations, because they need to be combined with the action of a spin-independent imaginary operator in order to correspond to an expectation value or magnetization density, as in Equations (0.12) or (0.14).

Löwdin [7] defined natural (spin) orbitals as the eigenfunctions of the full oneparticle density matrix in the molecular orbital basis. The NOs, NSOs, and the orbitals used to construct the current density, as described in this section, are not the same as Löwdin's, but they are based on related concepts. The restricted active space self consistent field (RASSCF) [8] and restricted active space state-interaction (RASSI) [9] programs of the Molcas suite [10] have for a long time included modules to calculate matrix elements of various kinds of operators in a basis of spin-free (SF) SR many-electron wavefunctions, which are subsequently transformed to the basis of SO wavefunctions. A key feature of this code is that it generates only the necessary SR density matrix information needed for matrix elements of (i) spin-free real operators (e.g. multipole moments, EFGs), (ii) spin-free real operators multi- 

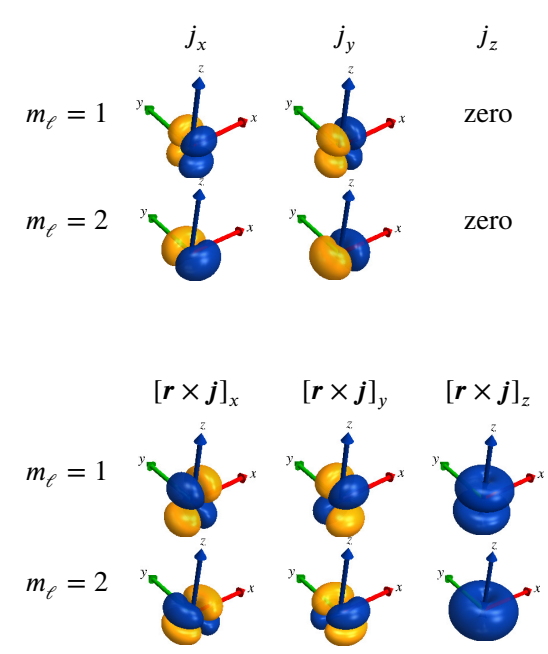

Fig. 0.1 Isosurfaces $( \pm 0.005 \mathrm{au})$ for the components of the paramagnetic current density $\boldsymbol{j}$ and the components of the orbital magnetization $m_{u}^{L}=[\boldsymbol{r} \times \boldsymbol{j}]_{u}$ generated from analytic expressions of the spherical harmonic angular functions $Y_{\ell}^{m_{\ell}}$ for $d$ orbitals, i.e. $\ell=2$ and $m_{\ell}=1$ to 2, multiplied with a normalized $3 d$ Slater-type radial function $r^{\ell \rightarrow 2} \exp (-\zeta r)$ with an exponent $\zeta=2$. There are no contributions from the $m_{\ell}=0 d_{\sigma}$ orbital. The isosurfaces for negative $m_{\ell}$ have the same shapes but the functions have the opposite sign patterns. The magnetization quantization axis is $z$.

plied by $\hat{S}_{x}, \hat{S}_{y}$, or $\hat{S}_{z}$ and the spin operators themselves, (iii) spin-free imaginary operators (e.g. orbital angular momentum components), or (iv) spin-free imaginary operators multiplied by $\hat{S}_{x}, \hat{S}_{y}$, or $\hat{S}_{z}$. We re-arranged this code in such a manner that the corresponding density matrices for cases (i) to (iv) are constructed explicitly for the SO wavefunctions first, while the contraction with the operator AO integral matrices is the very last step of the calculation. This approach gives access both to the operator matrix elements for the SO wavefunctions, and the related (single-state or transition-) density matrices and their eigenfunctions (orbitals). The NOs of this section are related to type (i). The NSOs are related to type (ii), and the eigenfunctions of $\mathbf{D}^{J}$ are related to type (iii), respectively.

Often, the magnetic properties of a metal complex are intimately tied to formally non-bonding $d$ or $f$ orbitals at the metal center (and magnetic couplings between metal centers, in multi-nuclear complexes or solids). The NOs and NSOs determined from ab-initio calulations then often appear as textbook examples of $d$ or $f$ orbitals, with varying degrees of covalency involving the ligands. In Section 0.3, we will not present individual orbital contributions to $m_{u}^{L}$. However, when the orbital angular momentum magnetization is caused by a single or a few non-bonding metal $d$ or $f$ orbitals, the resulting magnetization $m_{u}^{L}$ may easily reveal the underlying contributions from the components of the magnetization density $\boldsymbol{r} \times \boldsymbol{j}$ of individual orbitals. For reference, Figures 0.1 and 0.2 display iso-surfaces of the paramagnetic current density components, and the components of the magnetization density, for $d$ and $f$ orbitals with different $m_{\ell}$. 


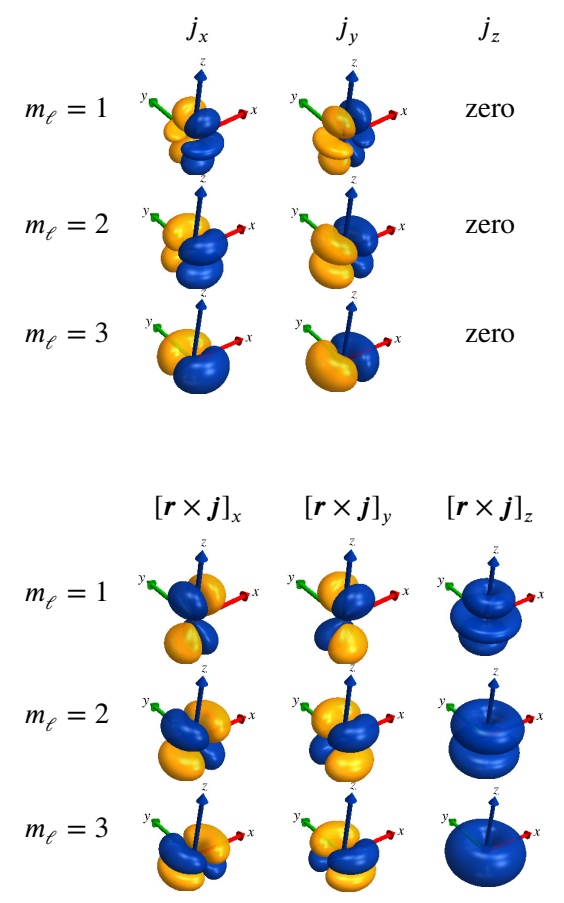

Fig. 0.2 Isosurfaces $( \pm 0.003 \mathrm{au})$ for the components of the paramagnetic current density $\boldsymbol{j}$ and the components of the orbital magnetization $m_{u}^{L}=[\boldsymbol{r} \times \boldsymbol{j}]_{u}$ generated from analytic expressions of the spherical harmonic angular functions $Y_{\ell}^{m_{\ell}}$ for $f$ orbitals, i.e. $\ell=3$ and $m_{\ell}=1$ to 3 , multiplied with a normalized $4 f$ Slater-type radial function $r^{\ell \rightarrow 3} \exp (-\zeta r)$ with an exponent $\zeta=2$. There are no contributions from the $m_{\ell}=0 f_{\sigma}$ orbital. The isosurfaces for negative $m_{\ell}$ have the same shapes but the functions have the opposite sign patterns. The magnetization quantization axis is $z$.

In the discussion in Section 0.3, the NOs and NSOs correspond to the usual real $d$ and $f$ orbitals. For a degenerate pair of these orbitals of $\pi, \delta$, or $\phi$ symmetry, an orbitally degenerate rotational symmetry-adapted doublet state arises if there is a single electron or a single hole in the level, i.e. for a combined population of 1 or 3 . The real NOs then have occupations of 0.5 or 1.5 each in the two electronic state components. As alluded to above, such an occupation pattern can be viewed as having formed complex linear combinations of the real NOs, with equal weight, to form angular momentum eigenfunctions with a given positive or negative $m_{\ell}$ value. (This is the reverse process of the textbook case where real atomic orbitals are formed from the complex eigenfunctions of $\hat{L}_{z}$.) In this case, after diagonalizing the magnetic moment operator in the basis of doublet state components, an un-quenched orbital angular momentum is usually obtained. A symmetry lowering that shifts the single occupation or hole dominantly or fully to one of the real NOs and breaks the orbital degeneracy of the state goes along with a quenching of the orbital angular momentum. 
All calculations discussed in Section 0.3 were carried out with the Molcas software, [10] employing the complete active-space self-consistent field (CASSCF) method and when necessary the CAS second-order perturbation theory (CASPT2) for the dynamic electron correlation, $[11,12]$ with ANO-RCC basis sets and the second-order Douglas-Kroll-Hess (DKH2) all-electron scalar relativistic Hamiltonian. [13] The SO interaction was treated with atomic mean-field integrals (AMFI) and the restricted active space state-interaction (RASSI) approach. [9] For brevity, the scalar relativistic (SR, i.e. pre-RASSI) and SO CASSCF and CASPT2 calculations are denoted as as SCF-SR, SCF-SO, PT2-SR, and PT2-SO in the following. Magnetic properties were calculated according to References 14, 5, 15 and 16. Single-crystal experimental structures or optimized structures (using density functional theory) were used for the calculations. Further details are provided in the relevant references where the calculations were published first. The calculations for $\mathrm{Co}(\mathrm{Tp})_{2}$ were carried out specifically for this work using similar computational details as those of Reference 17, with an active space comprising the $3 d$ and $3 d^{\prime}$ orbitals as well as two ligand orbitals of $\sigma$ symmetry $(\operatorname{CAS}(11,12))$. For FeLX complex, a CAS $(12,13)$ including the five $3 d$, the correlation $3 d^{\prime}$, and three bonding orbitals is used with CASPT2 energies. 5 quintets, 15 triplets and 10 singlets states are considered. $\left[\mathrm{Ni}(\mathrm{L})_{2}\left(\mathrm{NO}_{3}\right)\right]^{+}$is described using a $\mathrm{CAS}(12,12)$ including two bonding $e_{g}$ orbitals, the five $3 d$ and a second $3 d^{\prime}$ shell with CASPT2 energies [31] and 10 triplets and 15 singlets states. $\mathrm{NpF}_{6}$ is described by a $\operatorname{RAS}(37 / 18 ; 7 ; 18) \mathrm{SCF}$ calculation. RAS1 comprises the $182 p_{F}$ orbitals, RAS2 the seven $5 f_{N p}$ orbitals and RAS 3 the 18 antibonding/correlation counterparts of the orbitals of RAS1. 2 holes/particules are allowed in RAS1/3. SOC is calculated with CASPT2 energies [43] and 7 doublets states. The $\left[\mathrm{U}\left(\mathrm{Tp}^{\mathrm{Me} 2}\right)_{2}\right.$ (bipy)]I complex is described using a CAS(3,7) (5f orbitals) with CASPT 2 energies and 35 quartets and 40 doublets states. The NOs and NSOs and magnetization plots were generated according to References [10,18-20].

\subsection{Case Studies: From Transition Metals to Actinides}

\subsubsection{A Trigonal Cobalt(II) Complex: $\mathrm{Co}(T \mathrm{Tp})_{2}$}

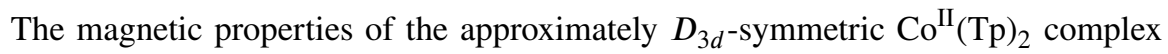
$(\mathrm{Tp}=$ trispyrazolylborate) were investigated by Tierney et al. using EPR and NMR spectroscopies. [21,22] Due to its interesting magnetic properties, we decided to perform calculations for this complex. The EPR measurements revealed a strong axial magnetic anisotropy characterized by $g_{\|}=8.48$ and $g_{\perp}=1.02$, with $g_{\|}$being in the direction of the three-fold principal axis of symmetry. The electronic structures of trigonal $\mathrm{Co}$ (II) complexes were rationalized theoretically already in the early 1950s by Abragram and Pryce [23], and then revisited by Jesson and McGarvey in 1970 s $[24,25]$. It is beneficial to consider the (approximate) $D_{3 d}$ point group symmetry of the complex as a trigonal distortion from an $O_{h}$ parent coordination. As 


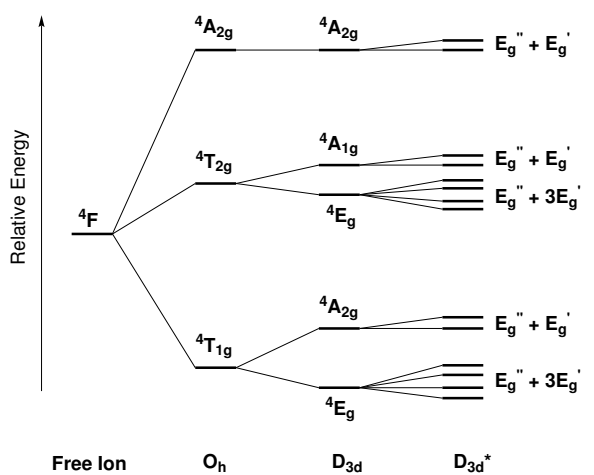

Fig. 0.3 Schematic energy diagram of the lowest electronic states deriving from the splitting of the ${ }^{4} F$ term of the $\mathrm{Co}^{2+}$ ion by an octahedral $\left(O_{h}\right)$ and a trigonal $\left(D_{3 d}\right)$ crystal-field. $D_{3 d}^{*}$ is the corresponding double group in which the SO states are classified.

seen in Figure 0.3, the $3 d^{7}$ configuration of the $\mathrm{Co}^{2+}$ ion leads to the spectroscopic $L S$-ground term ${ }^{4} F$. The excited ${ }^{4} P$ term is much higher in energy and therefore not discussed here. The seven-fold orbital degeneracy of the ground term is split by an octahedral ligand-field (LF) into the spin quartets ${ }^{4} T_{1 g},{ }^{4} T_{2 g}$ and ${ }^{4} A_{2 g}$. The $O_{h}$ ground state (GS) ${ }^{4} T_{1 g}$ is then split by the trigonal LF distortion into two spin quartets ${ }^{4} E_{g}$ and one spin quartet ${ }^{4} A_{2 g}$. The zero-field splitting (SO interaction) finally breaks the four-fold spin- and two-fold orbitally-degenerate SR GS into four Kramers doublets.

The electronic structure and the EPR $g$-factors of $\mathrm{Co}(\mathrm{Tp})_{2}$ were calculated at the CAS level, and the resulting $3 d$ NOs for the SO GS are shown in Figure 0.4, along with the orbital and spin magnetizations. At the SCF-SO level, the GS doublet derives almost exclusively from the SR GS ${ }^{4} E_{g}(98 \%)$. It is separated from the first excited state (ES) by $220 \mathrm{~cm}^{-1}$. As the EPR measurements were performed at 3.6 $\mathrm{K}$, only the GS Kramers doublet is thermally populated, and hence, the electronic $g$-factors can be rationalized with a pseudo-spin $\mathbb{S}=1 / 2$ and the following spin Hamiltonian:

$$
\hat{\mathcal{H}}_{S}=\mu_{B}\left[g_{\|} B_{\|} \hat{\mathbb{S}}_{\|}+2 g_{\perp}\left(B_{\perp} \hat{\mathbb{S}}_{\perp}\right)\right]
$$

The SCF-SO calculation gives for the GS Kramers doublet $g_{\|}=8.72$ and $g_{\perp}=1.01$. The large magnitude of the magnetic moment along the $\|$ magnetic axis results from a large unquenched orbital angular momentum, and from the reinforcement of $\left\langle L_{\|}\right\rangle$ and $\left\langle S_{\|}\right\rangle$(Figure 0.4). One can notice that the calculated spin expectation value of $\left\langle S_{\|}\right\rangle=1.44$ is very close to the expected value for a SR spin quartet $\langle S\rangle=3 / 2$, which is evidence of the rather weak mixing of SR states in the SO GS. Introduction of the dynamic correlation at the PT2-SO level does not improve the calculated $g$ factors $\left(g_{\|}=8.77\right.$ and $\left.g_{\perp}=0.84\right)$. The deviations from the experimental data are tentatively attributed to the dipolar spin-spin interactions, which are not included in our calculations but are known to contribute to the ZFS in transition metals [26], and 


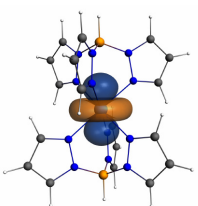

NOs: $a_{1 g}: 1.952$

NSOs: $a_{1 g}: 0.032$

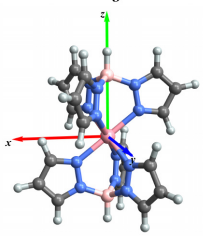

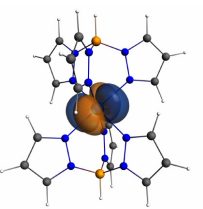

$\boldsymbol{e}_{\mathrm{g}}$ (1): 1.542

$e_{g}(1): 0.512$

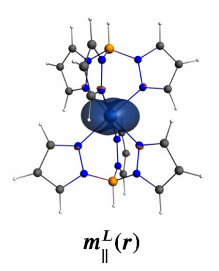

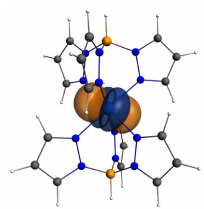

$\boldsymbol{e}_{g}(\mathbf{1}): 1.469$

$e_{g}(1): 0.444$

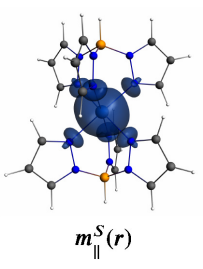

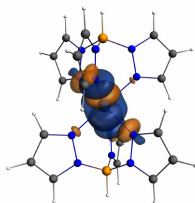

$e_{g}(2): 1.018$

$e_{g}(2): 0.945$

$\left\langle L_{\|}\right\rangle=1.48$

$\left\langle S_{\|}\right\rangle=1.44$

$g_{\text {Calc. }}=8.72$

$g_{\text {Expt. }}=8.48$

Fig. 0.4 Top row: Selected isosurfaces $( \pm 0.03 \mathrm{au})$ and occupations of the natural orbitals (NOs) of the SO GS of $\mathrm{Co}(\mathrm{Tp})_{2}$. The spin populations of the corresponding natural spin orbitals (NSOs) are also given for comparison. The NSOs isosurfaces appear to be very similar to those of the NOs and are therefore not shown. Bottom row: Orientation of the principal magnetic axes, isosurfaces $( \pm 0.001 \mathrm{au})$ of the orbital $\left(m_{\|}^{L}(\boldsymbol{r})\right)$ and spin $\left(m_{\|}^{S}(\boldsymbol{r})\right)$ magnetizations, and $g$-factors for the SO GS of $\mathrm{Co}(\mathrm{Tp})_{2}$. Doublet components with $\left\langle S_{\|}\right\rangle>0$. SCF-SO Results.

to the fact that the measurement was done for the solid state but the calculation is for the isolated complex.

The large magnitude of $\left\langle L_{\|}\right\rangle$and $\left\langle S_{\|}\right\rangle$in $\operatorname{Co}(\mathrm{Tp})_{2}$ can be related to the populations of the NOs and NSOs of the SO GS. The five-fold degeneracy of the $3 d$ orbitals is split by the trigonal CF into a non-degenerate $a_{1 g}$ (using $D_{3 d}$ symmetry labels), and two sets of degenerate $e_{g}$ orbitals. The $a_{1 g}$ orbital corresponds to the real $3 d_{z^{2}}$ orbital with $m_{\ell}=0$, whereas the $e_{g}$ orbitals correspond to real linear combinations of the complex $3 d$ orbital angular momentum eigenfunctions with $m_{\ell}= \pm 1$ and \pm 2 . [27] As seen in Figure 0.4, the deviation from a perfect $D_{3 d}$ symmetry in Co(Tp) $)_{2}$ leads to slightly inequivalent $e_{g}$ orbitals with different NO and NSO populations, but the symmetry breaking is relatively minor.

For an idealized $D_{3 d} \mathrm{Co}(\mathrm{Tp})_{2}$ complex, the wavefunction of the SR GS ${ }^{4} E_{g}$ would be an admixture of equal weight of the configurations (a) and (b) shown in Figure 0.5 . Due to the dominant contribution of the SR GS in the SO GS, the SO NO occupations (very close to $\left.a_{1 g}^{2} e_{g}(1)^{3} e_{g}(2)^{2}\right)$ arise directly from these two configurations. For an electronic state component with even weights of configurations (a) and (b), we would expect an occupation of 1.5 for each of the $e_{g}(1)$ metal orbitals. From this occupation scheme one further expects a theoretical $\left\langle S_{\|}\right\rangle$of 1.5 , which results from contributions due to both sets of $e_{g}$ orbitals. Indeed, the $a_{1 g}$ orbital is doubly occupied, and hence, does not contribute to the spin angular momentum. The corresponding NSO spin population (Figure 0.4) is close to zero, accordingly. The $e_{g}(1)$ set shares one unpaired electron and each orbital contributes to 0.5 to $2\left\langle S_{\|}\right\rangle$, whereas the $e_{g}(2)$ orbitals are both singly occupied and each contribute approximately to 1 

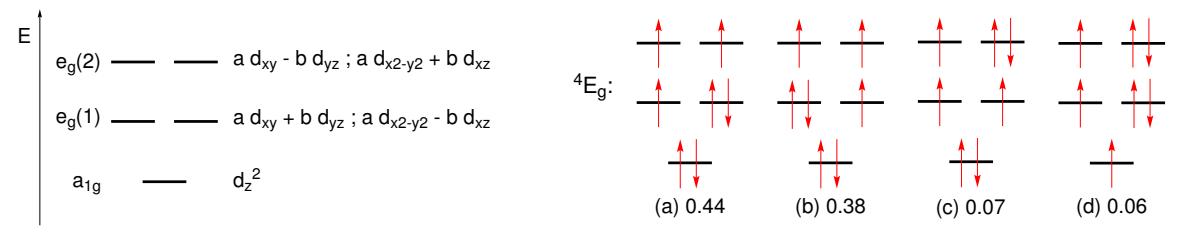

Fig. 0.5 Left: Splitting of the $3 d$ orbitals in the $D_{3 d}$ symmetry point group. Right: Composition of the SR ground state wavefunction ${ }^{4} E_{g}$. Only the configurations with a weight larger than $5 \%$ are shown.

to $2\left\langle S_{\|}\right\rangle$. These contributions from both $e_{g}$ sets give a tangerine-shaped $m_{\|}^{S}(\boldsymbol{r})$ isosurface at the cobalt center. The calculated $\left\langle S_{\|}\right\rangle=1.44$ results principally from this description. However, the slight symmetry breaking leads to a SR GS ${ }^{4} E_{g}$ with admixtures of additional configurations (c) and (d) of Figure 0.5. These configurations, in combination with the SO interaction, affect the populations of the $a_{1 g}$ and $e_{g}(1)$ NOs and NSOs. Furthermore, sizable spin-magnetization is also seen on the ligand-nitrogen atoms. This effect is associated with the $e_{g}(2)$ orbitals (see Figure 0.4 ), which are seen to afford $\mathrm{Co}-\mathrm{N}$ antibonding interactions. In reference to the orbital diagram of Figure 0.5 , the ligands donate electron density to the metal $e_{g}(2)$ shell, but only with $\downarrow$ spin because the $\uparrow$ spin metal $3 d$ orbitals are filled. This donation is reflected by NO populations of 1.018, slightly larger than the idealized value of 1 , and by NSO spin populations slightly below 1 due to the contributions from $\downarrow$ spin in the $e_{g}(2)$ shell.

$\left\langle L_{\|}\right\rangle$and the associated orbital magnetic moment results essentially only from contributions of the $e_{g}(1)$ orbitals. The $a_{1 g}$ orbital with $m_{\ell}=0$ does not contribute to $\left\langle L_{\|}\right\rangle$, whereas the contribution from the $e_{1 g}(2)$ orbitals to the orbital angular momentum is null. (Due to their single occupations, their contributions to $\left\langle L_{\|}\right\rangle$correspond to a sum of two $\hat{L}_{\|}$eigenfunctions, with eigenvalues $+m_{\ell}$ and $-m_{\ell}$.) For the $e_{g}(1)$ shell, the electronic occupation of ca. $3 / 2$ in each orbital allows to maximize their contributions to $\hat{L}_{\|}$. In this case, one can make a linear combination of the two real orbitals to obtain $\hat{L}_{\|}$eigenfunctions with $\pm m_{\ell}$, one of each being occupied in the two components of the degenerate GS. The composition of the $e_{g}(1)$ orbitals in terms of the real $3 d$ orbitals calculated for $\mathrm{Co}(\mathrm{Tp})_{2}$ is the following, with $z$ coinciding with the 3 -fold symmetry axis

$$
\begin{aligned}
& e_{1 g}(1)_{a}=-0.74 d_{x y}+0.35 d_{y z}+0.40 d_{x z}+0.42 d_{x 2-y 2} \\
& e_{1 g}(1)_{b}=0.44 d_{x y}+0.42 d_{y z}+0.33 d_{x z}+0.73 d_{x 2-y 2}
\end{aligned}
$$

The presence of the $x z$ and $y z$ contributions reflects the tilting of the two orbitals with respect to the $z$ axis, whereas the mixing of $x y$ and $x^{2}-y^{2}$ mainly simply reflects the arbitrary choice of the ligand azimuth positions. Using $e_{g}(1)_{a}$ and $e_{g}(1)_{b}$, one can make a linear combination that would lead to an orbital angular momentum expectation value of $\left\langle L_{\|}\right\rangle=1.73$. This linear combination is responsible for the oblate $m_{\|}^{L}(\boldsymbol{r})$ visible in Figure 0.4. Refer to Figure 0.1: The two orbitals in question 


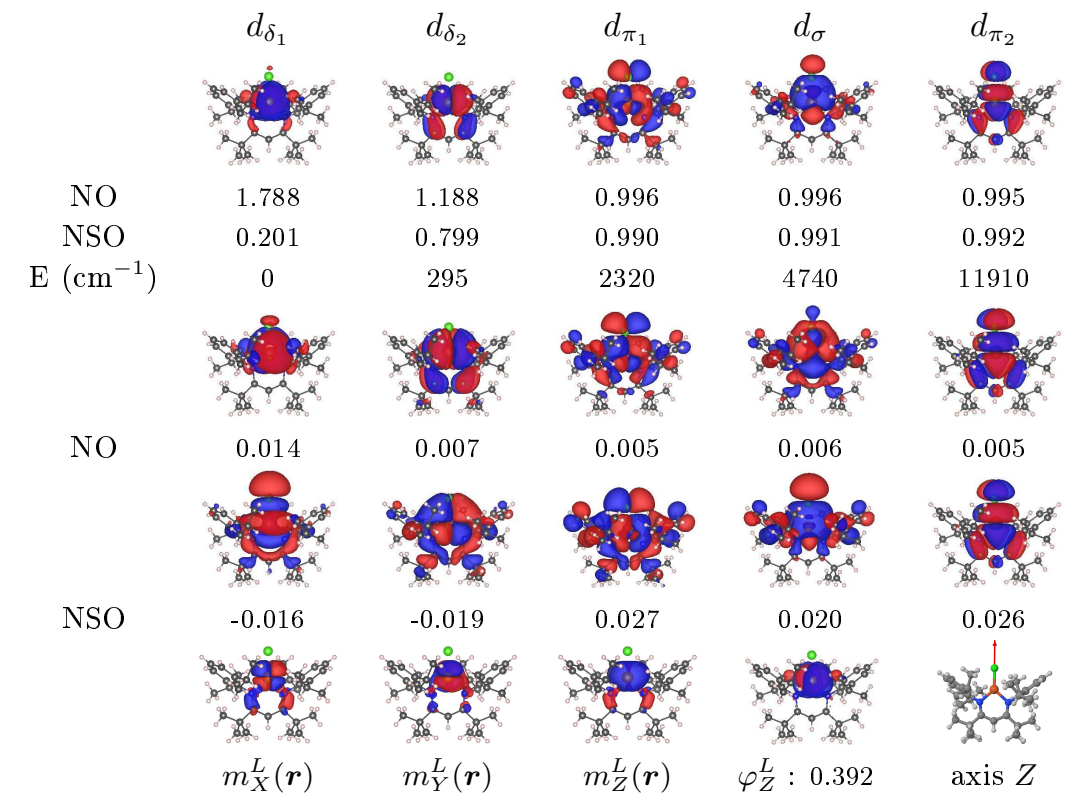

Fig. 0.6 Top row: Isosurface and occupation number of selected NOs for the two lowest SO states of [FeLCl]. The spin population of the corresponding NSOs along the magnetic axis and the energy of the corresponding canonical orbitals are given for comparison. Second row: Isosurface and occupation number of selected NOs. Third row: Isosurface and spin population of selected NSOs. Bottom row: Isosurface of the orbital magnetization $\left(m_{u}^{L}(\boldsymbol{r})\right)$ and of its principal current density natural orbital $\left(\varphi_{u}^{L}\right)$ along the magnetic axis. Isovalues: \pm 0.07 au for orbitals $; \pm 0.0001$ au for magnetization.

are approximately of $d_{\delta}\left(\left|m_{\ell}\right|=2\right)$ symmetry. The corresponding orbital magnetization for an $m_{\ell}= \pm 2$ angular momentum eigenfunction affords the 'doughnut' shape in the bottom right of the figure, which is quite similar to the actual calculated $m_{\|}^{L}$. The lowering of symmetry from linear to trigonal causes the model to deviate from the expected $\left\langle L_{\|}\right\rangle= \pm 2$ for a $d_{\delta}$ hole. The model value is not far from the CASSCF result of $\left\langle L_{\|}\right\rangle$1.48. The deviation between the model and the ab-initio data is also attributed to the slight geometrical distortions in $\mathrm{Co}(\mathrm{Tp})_{2}$, which decrease the magnitude of the orbital angular momentum further, relative to the model, via an uneven population of $e_{g}(1)_{a}$ and $e_{g}(1)_{b}$.

\subsubsection{A High-Spin Fe(II) Complex: FeLCl}

In the $[\mathrm{FeLCl}]$ complex with $\mathrm{L}=\beta$-diketiminate (the structure is shown in Figure 0.6 ), the $\mathrm{Fe}(\mathrm{II})$ center is in a $3 d^{6}$ high-spin configuration with a spin quintet ground state. EPR measurements in the $\mathrm{X}$ band reveal a quasidegenerate $M_{S}= \pm 2$ ground doublet with an axial $g=10.9$ and a small splitting of $\Delta=0.35 \mathrm{~cm}^{-1}$. The next 
component of the spin quintet is estimated to be higher than $150 \mathrm{~cm}^{-1}$ [28]. In a pure spin quintet, the $M_{S}= \pm 2$ components have a $g$-factor of 6 ; the experimental value shows that there is a large orbital contribution due an only partial quenching of the orbital moment and a low lying SR state. This is confirmed by CASPT2 calculations which show that the first excited state lies at $516 \mathrm{~cm}^{-1}$ above the GS. With the SO coupling, the spin quintet splits into five components by $0.34,118,132$ and 180 $\mathrm{cm}^{-1}$ [29]. The spin Hamiltonian is either written for the ground non KD with a small ZFS and a large anisotropic $g$ factor, or by a pseudo-spin $\mathbb{S}=5$ with a large ZFS splitting and a nearly isotropic $g$ tensor. The following analysis is performed based on the non KD perspective.

In a non $\mathrm{KD}$, only one component of the $g$ tensor is non-zero. In the [FeLCl] complex, the magnetic axis of the ground doublet is parallel to the $C_{2}$ symmetry axis of the molecule, which is taken to be along the $Z$ direction and coinciding with the quantization axis. The two $d_{\delta}$ orbitals are non-bonding with the chlorine atom and are the lowest in energy while the $d_{\pi}$ and $d_{\sigma}$ are anti-bonding and destabilized. The two $d_{\delta}$ are split by $300 \mathrm{~cm}^{-1}$ due to a differential interaction with the L ligand (cf Figure 0.6). The $d_{\pi_{2}}$ orbital is more anti-bonding than the $d_{\sigma}$ due to a strong $\pi$ interaction with the $\mathrm{L}$ ligand. The two low lying SR states correspond to configurations $\left(d_{\delta_{1}}\right)^{2} d_{\delta_{2}} d_{\pi_{1}} d_{\pi_{2}} d_{\sigma}$ and $d_{\delta_{1}}\left(d_{\delta_{2}}\right)^{2} d_{\pi_{1}} d_{\pi_{2}} d_{\sigma}$ respectively, and the orbital moment is quenched. These states are strongly coupled by SO coupling due to the small energy gap, leading to a mixing of $80 \%$ and $20 \%$; this strong mixing leads to the partial un-quenching of the orbital moment, to the large splitting of the spin quintet and to the large magnetic anisotropy of the ground doublet.

NOs and NSOs for the ground doublet are shown in Figure 0.6. While in a nonKramers doublet NOs' populations might be different, they are almost identical for the two components with 1.8 in $d_{\delta_{1}}$ and 1.2 in $d_{\delta_{2}}$ as expected from the SO coupling mixing. The mixing by $\mathrm{SO}$ coupling is much smaller for the other three components and the populations of $d_{\delta_{1}}$ and $d_{\delta_{2}}$ lie in the ranges 1.89-1.98 and 1.08-0.99 respectively. There is one correlation orbital per occupied $d$ orbital and the largest correlation is obtained for the $d_{\delta_{1}}$ orbital which is the most populated one.

In a non-Kramers doublet, NSOs are only defined along the magnetic axis. The spin population follows the electronic configuration: it appears as a complement to 2 to the electron occupancy of the orbitals. The correlation NSOs are shown in the 3rd row of Figure 0.6 and have a spin population of $\approx \pm 0.02$. There is a delocalization of the $\alpha$ spin density in the $\pi$ and $\sigma$ symmetries and a spin polarization with the appearance of a $\beta$ spin density on the ligands in the orbitals of $\delta$ symmetry. Finally, as shown in the last row of Figure 0.6, the magnetization is approximately cylindrical, in accordance with Fig. 0.1. Interestingly, the magnetization spreads out onto the lower part of the L ligand and exhibits a sign change, meaning that the covalent interactions between the metal and the ligand induce an orbital magnetization on the ligand that is of opposite sign to that on the metal. The orbital contributing the most to the orbital magnetization is $d_{\delta_{1}}$.

In this complex, the NOs and NSOs permit a visualization of the strong mixing of two SR states by So coupling which, by partially de-quenching the orbital magnetic moment, leads to a strong magnetic anisotropy. Furthermore, the 'secondary' 

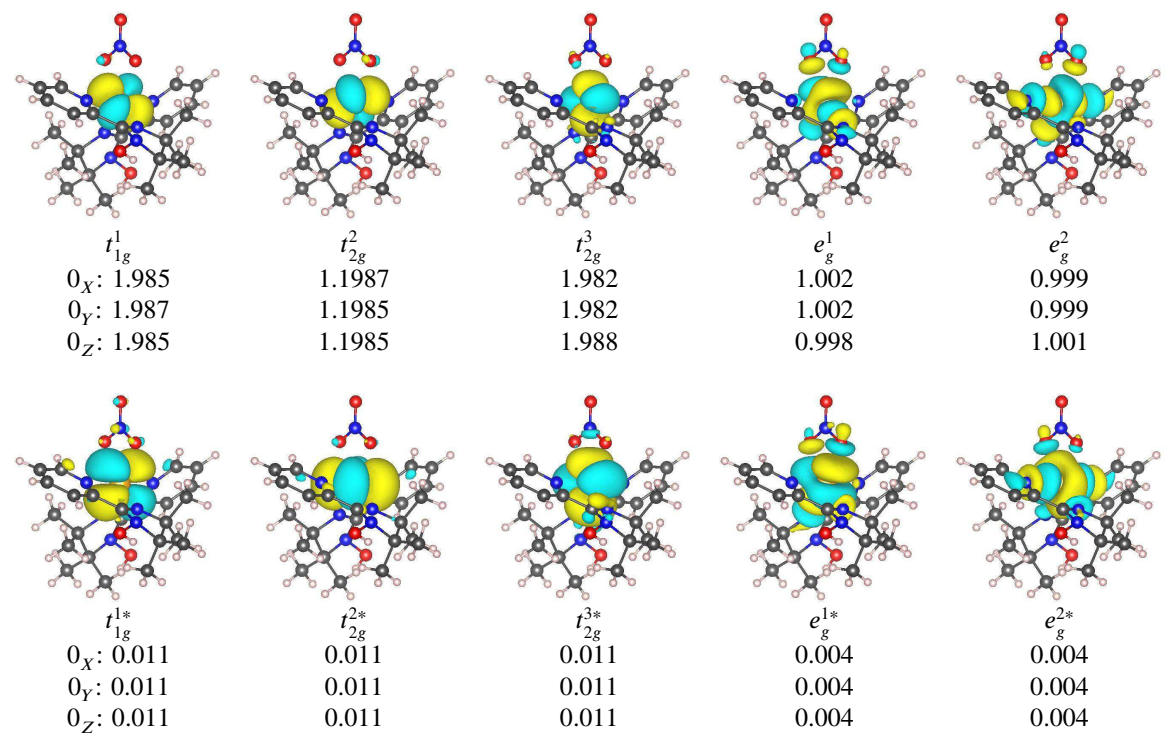

Fig. 0.7 Isosurfaces $( \pm 0.02 \mathrm{au})$ and occupation numbers for selected NOs of the three components $\left(0_{X}, 0_{Y}\right.$ and $\left.0_{Z}\right)$ issued from the GS triplet $\left[\mathrm{Ni}\left(\mathrm{HIM}_{2}-\mathrm{py}\right)_{2}\left(\mathrm{NO}_{3}\right)\right]^{+}$.

NOs and NSOs permit to quantify the effects of dynamical correlation and of spindelocalization and -polarization.

\subsubsection{Zero-Field Splitting in an octahedral Ni(II) complex}

The complex $\left[\mathrm{Ni}\left(\mathrm{HIM}_{2}-\mathrm{py}\right)_{2}\left(\mathrm{NO}_{3}\right)\right]^{+}$is a six-coordinate complex of $\mathrm{Ni}(\mathrm{II})$ where the two bidentate $\mathrm{HIM}_{2}$ - py ligands ((2-(2-pyridyl)-4,4,5,5-tetramethyl-4,5dihydro-1H-imidazolyl-1-hydroxy) and the nitrate ion form a deformed octahedral coordination (the structure is shown in Figure 0.8), sphere [30]. The SR ground state is a spin triplet arising from the $\left(t_{2 g}\right)^{6}\left(e_{g}\right)^{2}$ metal configuration. SO coupling with excited states, mostly a triplet arising from the $\left(t_{2 g}\right)^{5}\left(e_{g}\right)^{3}$ configuration, removes the degeneracy of the three components of the ground triplet, leading to zero-field splitting (ZFS). The magnetic anisotropy of the ground triplet may be characterized by a spin Hamiltonian whose canonical form is

$$
\begin{aligned}
\hat{\mathcal{H}}_{S}= & \mu_{B}\left(g_{X} B_{X} \hat{\mathbb{S}}_{X}+g_{Y} B_{Y} \hat{\mathbb{S}}_{Y}+g_{Z} B_{Z} \hat{\mathbb{S}}_{Z}\right) \\
& +D\left(\hat{\mathbb{S}}_{Z}^{2}-\frac{1}{3} \mathbb{S}(\mathbb{S}+1)\right)+E\left(\hat{\mathbb{S}}_{X}^{2}-\hat{\mathbb{S}}_{Y}^{2}\right)
\end{aligned}
$$

The ZFS parameters $D$ and $E$ and the three $g$ factors have been determined by HighField High-Frequency spectroscopy. $D=-10.1 \mathrm{~cm}^{-1}, E / D=0.24, g_{X}=g_{Y}=$ 
2.20 and $g_{Z}=2.27$. This is nicely reproduced by CASPT 2 calculations using a CAS $(13,13)$ including two bonding $e_{g}$ orbitals and a second $d$ shell [31].

As it is usually the case in transition metals, the anisotropic magnetic behavior is borne by the ZFS of the ground spin state, and the large negative $D$ value leads to an axial magnetization. The three components of the ground spin triplet are non degenerate and consequently, the expectation value of the magnetic moment vanishes. Magnetic properties arise from the coupling between the three components by the Zeeman operator; the closer the two components are in energy, the more magnetic the corresponding direction is. [29,31]

The two lowest components, denoted $0_{X}$ and $0_{Y}$, are close in energy by $1.8 \mathrm{~cm}^{-1}$ while the third one, $0_{Z}$, has a larger energy gap $12.1 \mathrm{~cm}^{-1}$. The NOs of the three components are depicted on Figure 0.7. They are very similar to each other, since the three components arise from the same spin triplet and the SO effects are small, but they differ slightly. The magnetic anisotropy arises precisely from these small differences. The negative $D$ value can be rationalized by the energetic ordering of the $d$ orbitals, and mostly from the splitting of the two $e_{g}$ orbitals which is due to the difference of $\sigma$ donation of the ligands to the metal [31]. Refering to Figure 0.7, the three $t_{2 g}$-like orbitals have a population that is slightly smaller than 2 due to the dynamic electron correlation. The two $e_{g}$-like orbitals have occupations close to 1 , one lower and one larger than 1. $e_{g}^{1}$ is the $d_{z^{2}}$ orbital and points towards two nitrogens of the $\mathrm{HIM}_{2}$ - py ligands while $e_{g}^{2}$ is the $d_{x y}$ and points towards the two other nitrogen atoms and the two oxygen atoms of the nitrate. Since the $\mathrm{HIM}_{2}$ - py ligands are better $\sigma$ donors than the nitrate, $e_{g}^{1}$ is more anti-bonding and more destabilized than $e_{g}^{2}$. As a consequence, in the two lowest $0_{X}$ and $0_{Y}$ components, the population of the most stabilized $e_{g}$ is larger than one while it is less than one for the $0_{Z}$ component. The population of the correlation $t_{2 g}^{*}$ NOs is about 0.01 each, which corresponds roughly to the depletion in the $t_{2 g}$ shell. The correlation in the $e_{g}$ shell is less important since the orbitals are singly occupied, and the population of the correlation $e_{g}^{*}$ NOs is slightly less than half those of the $t_{2 g}^{*}$ ones.

Magnetic properties arise from the coupling between two components. The coupling between $0_{X}$ and $0_{Y}$ is the largest since these components are the closest in energy. This coupling defines the direction of the axial magnetization, denoted $Z$. The NSOs for this quantization direction are shown in Figure 0.8 in comparison to those in directions $X$ and $Y$ obtained from the coupling between $0_{Y}$ and $0_{Z}$ and between $0_{X}$ and $0_{Z}$ respectively. The spin density is mostly borne by the $e_{g}$ orbitals with a spin population close to 1 , as expected, but it should be noted that one of the $e_{g}$ has a larger spin population, namely the $d_{U^{2}}$ when the axis is along $U=X, Y, Z$. Spin polarization appear with both $\alpha$ and $\beta$ densities and both through $\sigma$ ( $e_{g}$-type orbitals) and $\pi$ ( $t_{2 g}$-like orbitals) bonding schemes. The $\alpha$ and $\beta$ populations of the $t_{2 g}$ NSOs are both 0.038 . The $\beta$ density is more delocalized on the ligands leading to spin polarization through the $\pi$ bonding. There is a small anisotropy, since the orbital with the lobes perpendicular to the $U$ axis has the lowest $\beta$ density. For the eg-like correlation NSOs, the $\alpha$ spin density is slightly larger than the $\beta$ one $(0.015$ against 0.01 ), the $\alpha$ one being more delocalized on the ligands. This spin delocaliza- 


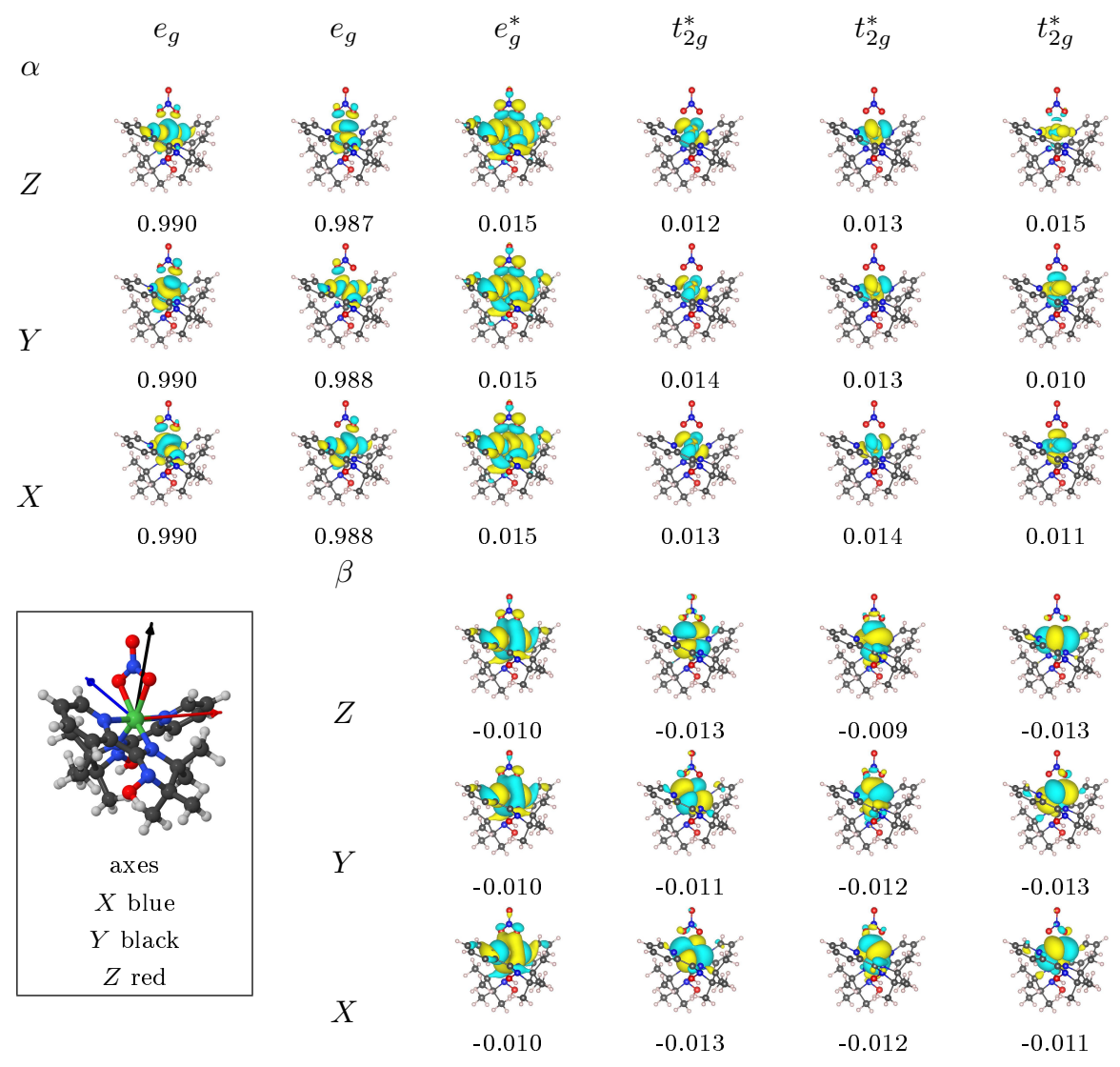

Fig. 0.8 Isosurfaces $( \pm 0.02 \mathrm{au})$ and spin populations for selected NSOs for the three components issued from the triplet $\mathrm{GS}$ of $\left[\mathrm{Ni}\left(\mathrm{HIM}_{2}-\mathrm{py}\right)_{2}\left(\mathrm{NO}_{3}\right)\right]^{+}$for the three principal magnetic axes $X, Y$ and $Z$ ).

tion through the $\sigma$ bonding is permitted by the half-filled $e_{g}$ shell while the $t_{2 g}$ shell is completly filled as it was the case for the $\mathrm{FeLCl}$ complex of .

The orbital magnetization density and orbital contributions along the three magnetic axes are shown on Figure 0.9. In this complex, the orbital moment is quenched in the GS as long as SO coupling is not considered. The SO coupling with the excited states gives rise to small orbital contributions which play a key role for the anisotropic properties. The largest contribution is along the $Z$ axis. The orbital magnetization in direction $U$ form a ring around this axis which spreads on the ligands perpendicular to this axis. The most contributing orbital is the $t_{2 g}$ one with the lobes perpendicular to the axis and to a lesser extend, the $e_{g}$ along the axis.

In this section, we have shown that when a pure spin triplet is split by the SO coupling with excited states, the NOs permit to quantify the small differences between the three components and to visualize the effects of correlation. The magnetic axes are defined by the coupling between two of the components arising fron the triplet 


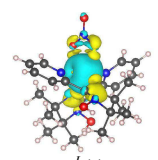

$m_{Z}^{L}(\boldsymbol{r})$

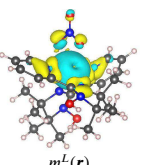

$m_{Y}^{L}(\boldsymbol{r})$

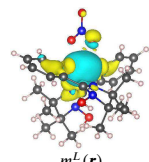

$m_{X}^{L}(\boldsymbol{r})$

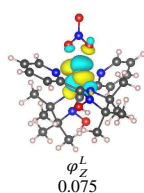

$\varphi_{Z}^{L}$
0.075

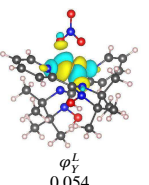

$\varphi_{Y}^{L}$
0.054

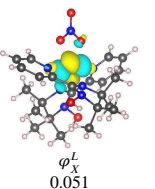

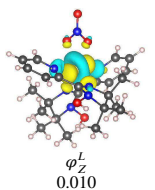

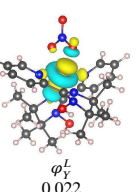

$\varphi_{Y}^{L}$
0.022

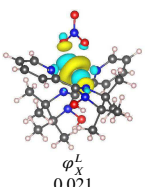

Fig. 0.9 Isosurfaces of the orbital magnetization $\left(m_{u}^{L}(\boldsymbol{r})\right)\left( \pm 5.10^{-5} \mathrm{au}\right)$ and of their principal current density natural orbitals $\left(\varphi_{u}^{L}\right)( \pm 0.02 \mathrm{au})$ for the three components issued from the triplet GS of $\left[\mathrm{Ni}\left(\mathrm{HIM}_{2}-\mathrm{py}\right)_{2}\left(\mathrm{NO}_{3}\right)\right]^{+}$along the three principal magnetic axes $X, Y$ and $Z$.

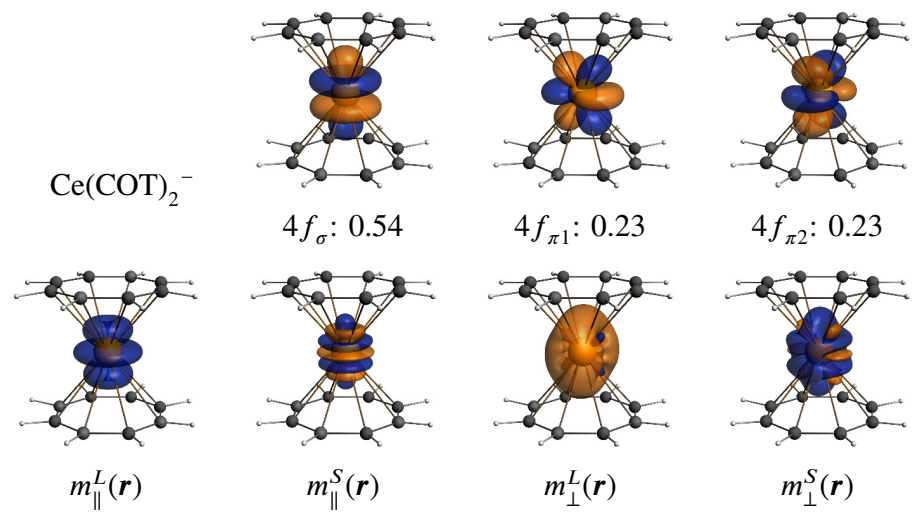

Fig. 0.10 Top row: Isosurfaces $( \pm 0.03 \mathrm{au})$ and occupations of selected natural orbitals of the SO $\mathrm{GS}$ of $\mathrm{Ce}(\mathrm{COT})_{2}{ }^{-}$. Bottom row: Isosurfaces $( \pm 0.001 \mathrm{au})$ of the orbital $\left(m_{u}^{L}(\boldsymbol{r})\right)$ and spin $\left(m_{u}^{S}(\boldsymbol{r})\right)$ magnetizations of $\mathrm{Ce}(\mathrm{COT})_{2}{ }^{-}$for the $\|$and $\perp$ magnetic axes. Doublet components with $\left\langle S_{u}\right\rangle>0$. SCF-SO results.

with their corresponding NSOs and orbital magnetization. It turns out that the spin density is slightly anisotropic and that there is some spin delocalization the $\sigma$ bonding scheme and spin polarization in the $\pi$ one. The orbital magnetization is a current around the considered axis and originates mainly from a $t_{2 g}$ like orbital. 


\subsubsection{Linear $\operatorname{Ln}\left(\right.$ III) Complexes: $\operatorname{Ln}(\mathrm{COT})_{2}^{-}$}

In $\mathrm{Ce}(\mathrm{COT})_{2}{ }^{-}\left(4 f^{1}\right.$ configuration), the linear crystal-field lifts the seven-fold degeneracy of the cerium $4 f$ orbitals to give rise to a $\mathrm{SR}^{2} \Sigma$ ground state when using labels for rotational symmetry, which is appropriate because $M_{\ell}$ for the $4 f$ shell is a good quantum number in the presence of an 8-fold symmetry axis. Introduction of the SO interaction mixes the $\uparrow$ and $\downarrow$ spin components of the SR GS and the excited ${ }^{2} \Pi$ state. The SO GS corresponds then to a KD of $M_{J}= \pm 1 / 2$ parentage which originates from the spectroscopic level ${ }^{2} F_{5 / 2}$ of the $\mathrm{Ce}^{3+}$ ion. The EPR $g$-factors associated with the GS were characterized experimentally by Walter et al. and reveled a planar magnetic anisotropy, with $g_{\|}=1.12$ and $g_{\perp}=2.27$. [32] These observed $g$-factors somewhat differ from the idealized values of $g_{\|}=0.86$ and $g_{\perp}=2.57$ for a $J=5 / 2, M_{J}= \pm 1 / 2$ ion micro-state. The deviations between the observed and $\mathrm{CF} g$-factors result from the different ratios of the $\mathrm{SR}^{2} \Sigma$ and ${ }^{2} \Pi$ states. This ratio is characterized in the GS wavefunction $|\psi\rangle$ by real coefficients $A$ and $B$, with $|\psi\rangle=A\left({ }^{2} \Sigma\right)+B\left({ }^{2} \Pi\right)$. For a $\mathrm{Ce}^{3+}$ ion micro-state, values of 0.65 and 0.76 would be expected for $A$ and $B$, respectively.

The electronic structure of $\mathrm{Ce}(\mathrm{COT})_{2}{ }^{-}$was calculated at the SCF-SO level with a minimal active space containing the seven $4 f$ orbitals. [19] The resulting natural orbitals of the SO GS are shown in Figure 0.10. As expected, the unpaired electron is shared between the $4 f_{\sigma}$ and the $4 f_{\pi}$ orbitals, with the occupation numbers corresponding to 0.74 and 0.68 for the real wavefunction coefficients $A$ and $B$, respectively. (For this effective one-electron system, the $\sigma$ and combined $\pi$ NO occupations are the squares of $A$ and $B$, respectively.) The calculated $g_{\|}=1.08$ and $g_{\perp}=2.35$ are in good agreement with the experiment. The planar magnetic anisotropy can be related to the orbital and spin magnetizations shown in Figure 0.10. Using Equations $0.2 \mathrm{a}$ and $0.2 \mathrm{~b}$, the orbital and spin magnetizations along the parallel magnetic axis of $\mathrm{Ce}(\mathrm{COT})_{2}{ }^{-}$are given as follows

$$
\begin{aligned}
m_{\|}^{S}(\boldsymbol{r}) & =\frac{1}{2}\left[A^{2} Y_{3}^{0} Y_{3}^{0}+B^{2} Y_{3}^{1} Y_{3}^{-1}\right] \\
& =\frac{A^{2}}{2} f_{\sigma}^{2}-\frac{B^{2}}{4}\left[f_{\pi+}^{2}+f_{\pi-}^{2}\right] \\
m_{\|}^{L}(\boldsymbol{r}) & =-B^{2} Y_{3}^{1} Y_{3}^{-1} \\
& =\frac{B^{2}}{2}\left[f_{\pi+}^{2}+f_{\pi-}^{2}\right]
\end{aligned}
$$

where $Y_{\ell}^{m_{\ell}}$ are spherical harmonics, and the $f_{\left|m_{\ell}\right| \pm}$ correspond to the $\ell=3$ tesseral harmonics which are real linear combinations of the complex $Y_{3}^{m_{\ell}}$ with the same $\left|m_{\ell}\right|$.

As seen in Eq. 0.19, the spin magnetization corresponds to positive contributions from the $f_{\sigma}^{2}$ density and to negative contributions from the $f_{\pi}^{2}$ densities, giving the alternating blue and orange lobes for $m_{\|}^{S}(\boldsymbol{r})$ in Figure 0.10 . The blue lobes represent the 
positive contributions from $f_{\sigma}$ and integrate to $A^{2} / 2=0.27$, while the orange lobes correspond to the contributions related to $f_{\pi}$ and integrate to $-2 B^{2} / 4=-0.23$. Therefore, the two contributions are almost equal and with opposite sign, leading to a very small spin expectation value $\left\langle S_{\|}\right\rangle=0.04$. This value is far from the expected value for a SR spin doublet $\langle S\rangle=1 / 2$ and reflects the importance of SO coupling in the $4 f$ shell. The orbital magnetization is simpler to analyze. Due to the lack of orbital angular momentum from the $4 f_{\sigma}$ orbital along the $\|$ magnetic axis, only the $4 f_{\pi}$ orbitals contribute to $m_{\|}^{L}(\boldsymbol{r})$. This is visible in Figure 0.10 where the orbital magnetization corresponds simply to a linear combination of each $f_{\pi}^{2}$ densities. The isosurface of $m_{\|}^{L}$ is also essentially identical to the idealized one for $m_{\ell}=1$ in Figure 0.2. Integration of the magnetization results an orbital angular momentum expectation value $\left\langle L_{\|}\right\rangle=0.46$. Overall, along the parallel axis the magnitude of the magnetic moment is driven by the orbital contribution from the $4 f_{\pi}$ orbitals. In turn, the occupation of these orbitals is a consequence of the SO interaction, mixing the $\mathrm{SR}^{2} \Sigma$ GS components with the ${ }^{2} \Pi$ state components of opposite spin projection. Overall, the electronic $g_{\|}$factor remains relatively small due to a quenching of the spin magnetic moment.

A similar approach can be used to analyze the magnetization densities along the perpendicular magnetic axis. As already mentioned in Section 0.2, the wavefunction components used for the analysis diagonalize the operator $\hat{L}_{\perp}+g_{e} \hat{S}_{\perp}$. In the model, this is achieved by taking a linear combination of the initial wavefunction $\left|\psi_{\|}\right\rangle$and of its Kramers conjugate $\left|\bar{\psi}_{\|}\right\rangle$:

$$
\begin{aligned}
& \left|\psi_{\|}\right\rangle=A Y_{3}^{0}-B \bar{Y}_{3}^{1} \\
& \left|\bar{\psi}_{\|}\right\rangle=A \bar{Y}_{3}^{0}-B Y_{3}^{-1}
\end{aligned}
$$

to give

$$
\left|\psi_{\perp}^{ \pm}\right\rangle=\frac{1}{\sqrt{2}}\left(\left|\psi_{\|}\right\rangle \pm\left|\bar{\psi}_{\|}\right\rangle\right)
$$

Substituting Equation 0.21 into Equations $0.2 \mathrm{a}$ and $0.2 \mathrm{~b}$, the orbital and spin magnetizations along the $\perp$ magnetic axis for the $\left\langle S_{\perp}\right\rangle>0$ component of the doublet can be written as:

$$
\begin{aligned}
m_{\perp}^{S}(\boldsymbol{r}) & =\frac{A^{2}}{2}\left[Y_{3}^{0} Y_{3}^{0}\right]-\frac{B^{2}}{4}\left[Y_{3}^{1} Y_{3}^{1}+Y_{3}^{-1} Y_{3}^{-1}\right] \\
& =\frac{A^{2}}{2} f_{\sigma}^{2}-\frac{B^{2}}{4}\left[f_{\pi-}^{2}-f_{\pi+}^{2}\right] \\
m_{\perp}^{L}(\boldsymbol{r}) & =-A B \sqrt{3}\left[Y_{3}^{0} Y_{3}^{0}\right]+\frac{A B \sqrt{3}}{2}\left[2 \cdot Y_{3}^{1} Y_{3}^{-1}+Y_{3}^{1} Y_{3}^{1}+Y_{3}^{-1} Y_{3}^{-1}\right] \\
& =-A B \sqrt{3}\left[f_{\sigma}^{2}+f_{\pi+}^{2}\right]
\end{aligned}
$$




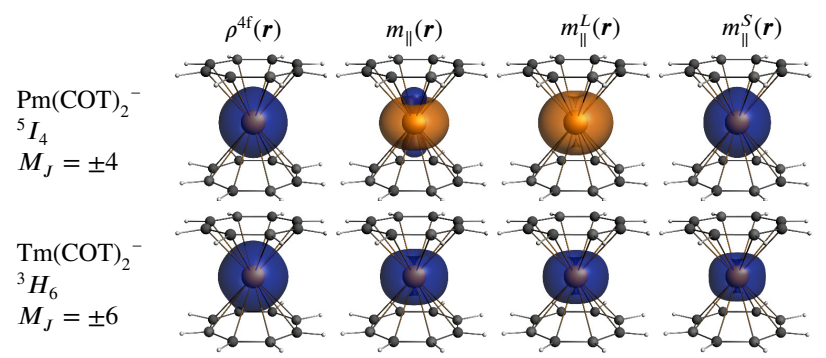

Fig. 0.11 Comparison of the ab-initio $4 f$ electron density $\left(\rho^{4 \mathrm{f}}(\boldsymbol{r})\right)$, orbital $\left(m_{\|}^{L}(\boldsymbol{r})\right), \operatorname{spin}\left(m_{\|}^{L}(\boldsymbol{r})\right)$ and total $\left.m_{\|}(\boldsymbol{r})\right)$ magnetizations for the SO GS of $\mathrm{Pm}(\mathrm{COT})_{2}{ }^{-}$and Tm(COT) ${ }_{2}{ }^{-}$. Doublet components with $\left\langle S_{\|}\right\rangle>0$. SCF-SO Results. Isosurfaces at \pm 0.001 au.

The isosurfaces of the corresponding magnetizations calculated $a b$-initio are shown in Figure 0.10. The spin magnetization corresponds to a dominant positive (blue isosurface) contribution which arises from the addition of the positive contributions of the $f_{\sigma}^{2}$ and $f_{\pi+}^{2}$ densities, which integrates to $0.38\left(A^{2} / 2+B^{2} / 4\right)$. This positive contribution is counterbalanced by a smaller negative contribution integrating to -0.11 . The negative contribution is characterized by the orange lobes in $m_{\perp}^{S}(\boldsymbol{r})$ and results from the $f_{\pi-}^{2}$ density. As seen in Eq. in $0.22 \mathrm{~b}$, the orbital magnetization results from the reinforcing contributions of the $f_{\sigma}^{2}$ and $f_{\pi+}^{2}$ densities. This leads to a large calculated expectation value for the orbital angular momentum $\left\langle L_{\perp}\right\rangle=-1.72$, characterized in Figure 0.10 by a large orange lobe. Therefore, the origin of the planar magnetic anisotropy in $\mathrm{Ce}(\mathrm{COT})_{2}{ }^{-}$resides in the large magnitude of the orbital magnetization for a field along the perpendicular magnetic axis, which is only to a small extent canceled by the spin magnetization.

Of particular interest are the relationships that may exist between the electron density generated by the $4 f$ orbitals $\left(\rho^{4 \mathrm{f}}(\boldsymbol{r})\right)$ and the orbital and spin magnetizations, and hence the magnetic moments. Comparisons of $\rho^{4 \mathrm{f}}(\boldsymbol{r})$ and of $m_{\|}^{L / S}(\boldsymbol{r})$ calculated for the SO GSs of Pm(COT $)_{2}{ }^{-}$and Tm(COT $)_{2}{ }^{-}$are provided in Figure 0.11. [19]

It is clear that the orbital and spin magnetization are only indirectly related to the $4 f$ electron density, because the electron density itself contains no information about the magnetic properties. In the case of $4 f^{n}$ complexes with $n \leq 7$, the spin magnetization tends to resemble the electron density. For instance, in $\mathrm{Pm}(\mathrm{COT})_{2}{ }^{-}$ $\left(4 f^{4}\right.$ configuration), both $\rho^{4 \mathrm{f}}(\boldsymbol{r})$ and $m_{\|}^{S}(\boldsymbol{r})$ have a prolate shape due to similar NO occupations and NSO spin populations, respectively. Here the 4 unpaired electrons are equally shared among the $4 f_{\sigma}, 4 f_{\pi}, 4 f_{\delta}$ and $4 f_{\phi}$ NOs (or NSOs).

On the other hand, $m_{\|}^{S}(\boldsymbol{r})$ tends to represent the unpaired electron(s), or the electron hole(s), in the case of $4 f^{n}$ systems with $n>7$. For example, the electronic occupation of the SO GS of Tm(COT) ${ }_{2}{ }^{-}\left(4 f^{12}\right.$ configuration) is $4 f_{\sigma}^{2} 4 f_{\pi}^{2} 4 f_{\delta}^{3} 4 f_{\phi}^{3}$, which leads to a mainly prolate $4 f$ electron density due to the lack of occupations in the $\delta$ and $\phi$ orbitals. The spin magnetization is, however, oblate because the two unpaired spins are associated with the $4 f_{\delta}$ and $4 f_{\phi}$ orbitals. Similarly to the spin mag- 
netization, $\rho^{4 \mathrm{f}}(\boldsymbol{r})$ is not directly related to the orbital magnetization. For instance, the very similar $4 f$ electron densities calculated for the GS of $\mathrm{Pm}(\mathrm{COT})_{2}{ }^{-}$and $\operatorname{Tm}(\mathrm{COT})_{2}{ }^{-}$lead to drastically different orbital magnetizations. $\operatorname{In} \operatorname{Pm}(\mathrm{COT})_{2}{ }^{-}$, a relatively large oblate $m_{\|}^{L}(\boldsymbol{r})$ with opposite sign to $m_{\|}^{S}(\boldsymbol{r})$ is calculated. The associated expectation values $\left\langle L_{\|}\right\rangle=-5.73$ and $\left\langle S_{\|}\right\rangle=1.73$ leads to a GS with a sizable magnetic anisotropy with $g_{\|}=4.50$ and $g_{\perp}=0.00$. $\operatorname{In~} \operatorname{Tm}(\mathrm{COT})_{2}{ }^{-}$, a positive oblate $m_{\|}^{L}(\boldsymbol{r})$ is calculated with $\left\langle L_{\|}\right\rangle=5.00$. As for all the complexes of the second half of the series, the orbital and spin magnetization reinforce each other in $\mathrm{Tm}(\mathrm{COT})_{2}{ }^{-}$to give an extremely large magnetic anisotropy with $g_{\|}=13.98$ and $g_{\perp}=0.00$.

The large orbital angular momenta in $\mathrm{Pm}(\mathrm{COT})_{2}{ }^{-}$and $\mathrm{Tm}(\mathrm{COT})_{2}{ }^{-}$can be rationalized with the help of the electronic occupation of the $4 f$ orbitals. As already mentioned, when a single electron or a single hole is equally shared in degenerate orbitals with the same $\left|m_{\ell}\right|$, one can generate a linear combination of the two orbitals which is an $\hat{L}_{\|}$eigenfunction with the eigenvalue $\pm m_{\ell}$, and hence maximize the contribution to the orbital angular momentum. For example, the two unpaired electrons in $\mathrm{Tm}(\mathrm{COT})_{2}{ }^{-}$are equally shared in the $4 f_{\delta}$ and $4 f_{\phi}$ orbitals such taht the orbital angular momentum is simply the sum of the two $m_{\ell}= \pm 2$ and \pm 3 .

The largest orbital angular momentum for an $f$-element in an axial environment would therefore be associated with $\sigma^{n} \pi^{1} \delta^{1} \phi^{1}$ and $\sigma^{n} \pi^{3} \delta^{3} \phi^{3}$ configurations. For the former case, the $f$-shell is less than half filled and the spin and orbital angular momenta would not reinforce each other. Accordingly, the latter case would lead to the overall largest magnetic moment (with $\sigma^{1}$ maximizing the spin magnetic moment). Such configurations are potentially accessible in linear environments with the $\mathrm{Dy}^{3+}$ and $\mathrm{Er}^{3+}$ ions, leading to a $\left|J=15 / 2, M_{J}= \pm 15 / 2\right\rangle$ Kramers doublet GS, and with the $\mathrm{Ho}^{3+}$ ion leading to a non-Kramers doublet $\left|J=8, M_{J}= \pm 8\right\rangle$. [33] The $|15 / 2, \pm 15 / 2\rangle$ doublet was characterized both experimentally and theoretically for the $\operatorname{Er}(\mathrm{COT})_{2}{ }^{-}$complex, [19,34] whereas the pentagonal-bipyramidal $\left.\left[\mathrm{Ho}\left(\mathrm{CyPh}_{2} \mathrm{PO}\right)_{2}\left(\mathrm{H}_{2} \mathrm{O}\right)_{5}\right)\right] \mathrm{I}_{3}$ complex affords an almost perfect $M_{J}= \pm 8 \mathrm{GS}$, therefore being very close to the ceiling magnetic moment for a single $f$ ion. [35,36]

To obtain larger magnetic moments, one would need to reach the even larger orbital angular momenta $m_{\ell}= \pm 4$ and \pm 5 associated with $g$ - and $h$-shells, couple orbital angular momenta from different centers, and/or create a large number of strongly coupled unpaired spins. Instead of trying to access exotic elements with partially filled shells of $\ell \geq 4$, [37] one could potentially use small metal clusters to generate magnetic superatoms. [38] For instance, transition metal clusters such as $\mathrm{Pb}_{12}{ }^{2-}, \mathrm{Sn}_{12}{ }^{2-}$ or the doped versions $\left[\mathrm{M} @ \mathrm{~Pb}_{12}{ }^{q}\right](\mathrm{M}=\mathrm{U}, \mathrm{Pu}, \mathrm{Am}, \mathrm{Cm})$, exhibit superatomic molecular orbitals that resemble atomic $g$ and $h$ orbitals. [39] The partial filling of these superatomic orbitals could lead to potentially gigantic orbital angular momenta. However, Hund's rules do not necessarily apply to unsupported metallic clusters because of potentially large Jahn-Teller distortions, which may break the high degeneracy and favor complexes with quenched magnetic moments. [40] 


\subsubsection{An Octahedral Np(VI) Complex: $\mathrm{NpF}_{6}$}

$\mathrm{NpF}_{6}$ crystallizes as a molecular crystal; the molecule is octahedral with a $5 f^{1}$ configuration. Its magnetic properties have been characterized by EPR and magnetic susceptibility measurements. [41,42] The ground state is a KD with a negative $g$ factor of -0.604 . This is well reproduced by CASPT2 calculations using a CAS(13/13) including the $5 f$ of the actinide and six $2 p$ orbitals of the ligands. [43]

In $\mathcal{O}_{h}$ symmetry, the seven $5 f$ orbitals split in three sets: the non-bonding and non-degenerate $f_{x y z}$ orbital belonging to the irreducible representation (irrep) $a_{2 u}$, the three $\pi$ antibonding $f_{x y}, f_{x z}$ and $f_{y z}$ orbitals of irrep $t_{2 u}$, and the three $\sigma$ antibonding $f_{x}, f_{y}$ and $f_{z}$ orbitals of irrep $t_{1 u}$. The ground state of the molecule is a KD of symmetry $E_{5 / 2}$ corresponding to a single electron in a $e_{5 / 2}$ spinor, which is a mixture of the $a_{2 u}$ and $t_{2 u}$ orbitals under the SO coupling interaction. The two components of the KD are, for a quantization axis along $z$

$$
\begin{aligned}
& |\psi\rangle=a\left|f_{x y z} ; \alpha\right\rangle+b \frac{1}{\sqrt{3}}\left(\left|f_{x y} ; \alpha\right\rangle+\left|f_{x z} ; \beta\right\rangle+i\left|f_{y z} ; \beta\right\rangle\right) \\
& |\bar{\psi}\rangle=a\left|f_{x y z} ; \beta\right\rangle+b \frac{1}{\sqrt{3}}\left(\left|f_{x y} ; \beta\right\rangle-\left|f_{x z} ; \alpha\right\rangle+i\left|f_{y z} ; \alpha\right\rangle\right)
\end{aligned}
$$

where $a$ and $b$ are real coefficients depending of on the crystal-field splitting between the $a_{2 u}$ and $t_{2 u}$ orbitals and the SO coupling When the quantization axis is along $x$ or $y$, the Kramers partners are obtained from Equations 0.23 by circular permutation $x \rightarrow y \rightarrow z$ and $x \rightarrow z \rightarrow y$ respectively. Without SO coupling, the ground state is orbitally non-degenerate and the orbital moment is quenched. According to Equation 0.23 , there are three contributions to the $g$ factor: i) a spin contribution $2-4 / 3 b^{2}$; the deviation from 2 arises from the admixture of a $\beta$-spin component through SO coupling, ii) a first order orbital angular momentum contribution $-8 / \sqrt{3} a b$ due to the partial de-quenching of the angular momentum by SO coupling iii) a second order orbital angular momentum contribution $2 / 3 b^{2}$ corresponding to the contribution of the $t_{2 u}$ orbitals.

The NOs of the ground KD are shown in Figure 0.12. As expected from Equation 0.23 , the $f_{x y z}$ and $f_{x y}, f_{x z}$ and $f_{y z}$ are partially occupied with respective populations of $a^{2}=0.74$ and a combined population of $b^{2}=0.28$. The dynamical correlation occurs equivalently in all the irreps either $g$ or $u$ with a depletion in the bonding orbital in favor of the corresponding antibonding one. The NSOs along $z$ axis with the largest spin population are in accordance with Equation 0.23: an $\alpha$ spin population of $a^{2}$ in orbital $f_{x y z}$ and $1 / 3 b^{2}$ in orbital $f_{x y}$ and a $\beta$ population of $1 / 3 b^{2}$ in orbitals $f_{y z}$ and $f_{z x}$. Spin correlation is the largest through $t_{1 u}$ orbitals with a $\alpha$ spin population in the metallic $t_{1 u}$ orbitals in favor of a $\beta$ population in the corresponding orbitals $t_{1 u}^{*}$ mostly localized on the ligands. Spin correlation occurs as well in the $g$ orbitals but to a lesser extent.The spin polarization appears clearly on the total spin magnetization with some $\beta$ spin density on all fluorine atoms. Spin densities for a magnetic field applied along another axis are obtained by circular permutations. 
As expected from the analysis of the contributions to the $g$ factor, there is an important contribution from orbital magnetization. The orbital magnetization for the field along $z$ is cylindrical around $z$ and resembles the $m_{\ell}=2$ 'double doughnut' shape in Figure 0.2, i.e. $f_{\delta}$ with respect to the quantization axis, but there is also a signature of magnetization contributions from $f_{\pi}\left(\left|m_{\ell}\right|=2\right)$ visible. The orbital magnetization shape isosurface is consistent with the qualitative analysis of the wavefunction, and - like the orbitals contributing to $m_{z}^{S}$ - it shows contributions from the fluorine ligands.

In this case, NOs and NSOs permit the visualization of electron and spin densities according to the wave function deduced from crystal field theory. Furthermore, they permit to 'see' the spin polarization on the fluorine ligands as well as an extent of magnetic coupling between the metal and the ligands that shows up in the orbital magnetization.

\subsubsection{Trigonal $U(I V)$ Complexes: $U C p_{3}-X$}

The electronic structure of $\left(\mathrm{C}_{5} \mathrm{Me}_{4} \mathrm{H}\right)_{3} \mathrm{UNO}\left(5 f^{2}\right.$ configuration) was rationalized with the help of KS-DFT and wavefunction calculations. [44] This complex represents one of the few examples of $\mathrm{U}^{\mathrm{IV}}$ complexes that exhibit temperatureindependent paramagnetism (TIP) at room temperature [45]; usually, the onset of temperature-dependent magnetism occurs at much lower temperatures. For $\left(\mathrm{C}_{5} \mathrm{Me}_{4} \mathrm{H}\right)_{3} \mathrm{UNO}$, the SR GS corresponds to the non-degenerate singlet state ${ }^{1} A_{1}$ in the $C_{3 v}$ symmetry point group, with the lowest SR triplet state lying above the GS at $3746 \mathrm{~cm}^{-1}$. The natural orbitals of the SR GS calculated at the CAS $(4,9)$ SCF-SO level are shown in Figure 0.13. The NOs from the SO calculations are very similar, and therefore not shown, while the occupations for the SO case, also shown in Figure 0.13, differ from the SR calculation.

The electronic structure of $\left(\mathrm{C}_{5} \mathrm{Me}_{4} \mathrm{H}\right)_{3} \mathrm{UNO}$ results from strong bonding and antibonding interactions between singly occupied $5 f_{\pi}$ orbitals of the $\mathrm{U}\left(\mathrm{C}_{5} \mathrm{Me}_{4} \mathrm{H}\right)_{3}$ fragment with the two singly occupied $\pi^{*}$ orbitals of the nitrosyl ligand. The bonding combinations correspond to the HOMO and HOMO-1 of the complex. As seen in Figure 0.13 , the occupations of these bonding $\pi$ orbitals differ significantly from 2. Indeed, the SR GS is strongly multi-configurational in character and can not be simply described just by the $\left|\pi_{1}^{2} \pi_{2}^{2} \pi_{1}^{* 0} \pi_{2}^{* 0}\right|$ 'DFT configuration'. In fact, this configuration represents only $14 \%$ of the GS wavefunction. The major configuration (20\%) corresponds to a double excitation among the $\pi$ orbitals $\left(\left|\pi_{1}^{\uparrow} \pi_{2}^{\uparrow} \pi_{1}^{* \downarrow} \pi_{2}^{* \downarrow}\right|\right)$, while configurations corresponding to single excitations among the $\pi$ orbitals also have sizable weights. This strongly correlated wavefunction leads to a GS with an effective U-N bond order (EBO) of 1.3 instead of 2 for the $\mathrm{U}-\mathrm{NO} \pi$ bond, as determined qualitatively from the occupations of the bonding vs. antibonding NOs. Introduction of the SO coupling mixes the SR GS with the lowest excited SR triplet states, leading to a non-degenerate GS with ca. 35\% of SR spin triplet character. This admixture leads to a sizable decrease in the occupations of the $\pi$ (and to a lesser degree of the $\pi^{*}$ ) 
NOs. This goes along with an increase of the occupation numbers of the $5 f_{\delta}$ orbitals, which arise formally from the SO coupling between the $5 f_{\pi}$ and the $5 f_{\delta}$ orbitals at the metal center, shifting electron density at the metal from $f_{\pi}$ to $f_{\delta}$. Since the $5 f_{\delta}$ are non-bonding, the EBO is slightly reduced by SO coupling, to below 1.2.

The calculated magnetic susceptibility $\chi$ for $\left(\mathrm{C}_{5} \mathrm{Me}_{4} \mathrm{H}\right)_{3} \mathrm{UNO}$ was calculated using the Van Vleck equation. The result is shown in Figure 0.14-(a). The agreement with the experiment (not shown) is satisfactory. [44] The reader is reminded that the magnitude of $\chi$ according to the Van Vleck equation depends on two terms for each electronic state, namely the Curie term and a linear response (LR) term. The former is explicitly temperature dependent $(1 / T)$ and is present for degenerate magnetic states, whereas the latter is temperature-independent and arises from contributions due to the magnetic coupling between different electronic states. [46] A more complicated behavior of $\chi$ may arise via a $T$-dependent Boltzmann LR and Curie terms average of the low-energy electronic state. The calculated $\chi T$ for $\left(\mathrm{C}_{5} \mathrm{Me}_{4} \mathrm{H}\right)_{3} \mathrm{UNO}$ reveals a linear increase with temperature, i.e. the susceptibility itself is constant. This TIP over such a large temperature range is due to the large energy gap (calculated as being over $3000 \mathrm{~cm}^{-1}$ ) between the non-degenerate SO GS and the first excited magnetic doublets. At room temperature, the lowest magnetic ESs are not populated to a significant degree, and therefore only the LR term for the GS contributes of the magnetic susceptibility.

The replacement of the axial nitrosyl ligand in $\left(\mathrm{C}_{5} \mathrm{Me}_{4} \mathrm{H}\right)_{3} \mathrm{UNO}$ by a methyl in $\left(\mathrm{C}_{5} \mathrm{H}_{5}\right)_{3} \mathrm{UCH}_{3}$ leads to the formation of a $\sigma$ bond between a fragment orbital of the $\mathrm{CH}_{3}$ radical ligand and a (mainly) $6 d_{\sigma} / 6 p_{\sigma}$ hybrid orbital of the $\left(\mathrm{C}_{5} \mathrm{H}_{5}\right)_{3} \mathrm{U}$ fragment. The bonding combination is formally doubly occupied, whereas the antibonding one is vacant. As seen in the right panel of Figure 0.13 , the $5 f$ orbitals do not strongly interact with the $\mathrm{CH}_{3}$ orbitals and remain principally non-bonding. Therefore, the SR GS corresponds to an orbitally degenerate spin triplet ${ }^{3} E$ in which the two unpaired electrons occupy mainly the $5 f_{a 1}$ and the $5 f_{e}$ orbitals. The $5 f_{\pi}$ and $5 f_{\delta}$ orbitals of the $C_{\infty h}$ point group belong to the same irreducible representation $(e)$ in the $C_{3 v}$ symmetry, leading to $5 f_{e}$ orbitals which are linear combinations of $5 f_{\pi}$ and $5 f_{\delta}$. The SO interaction mixes the SR GS with the lowest excited SR triplet states and gives a non-degenerate GS. Due to the strong mixing of states, the SO GS only contains 14 weight $\%$ of the SR GS, and derives principally (42\%) from the lowest SR ES ${ }^{3} A_{1}$. Thus, the SO occupation numbers of the non-bonding NOs differ significantly from the ones calculated for the SR GS. As for the nitrosyl complex, the SR and SO NOs of $\left(\mathrm{C}_{5} \mathrm{H}_{5}\right)_{3} \mathrm{UCH}_{3}$ themselves are very similar, and only the SR set is shown in Figure 0.13 .

The calculated $\chi T$ for $\left(\mathrm{C}_{5} \mathrm{H}_{5}\right)_{3} \mathrm{UCH}_{3}$ is shown in Figure 0.14-(a) and reveals a very different magnetic behavior than $\left(\mathrm{C}_{5} \mathrm{Me}_{4} \mathrm{H}\right)_{3} \mathrm{UNO}$. At low $T, \chi T$ increases linearly with $T$. This is the TIP regime and it is the result of the magnetic coupling that takes place between the non-degenerate GS and the two lowest magnetic ESs. These ESs are calculated lying at 192 and $347 \mathrm{~cm}^{-1}$ above the GS and are therefore not populated at low $T$. Above ca. $60 \mathrm{~K}$, however, the thermal population of the lowest ESs becomes non-negligible. The Curie term contributions to the magnetic susceptibility increase and $\chi$ becomes temperature-dependent. This behavior is typical for 
U(IV) complexes. [47] The magnetic behavior of $\left(\mathrm{C}_{5} \mathrm{H}_{5}\right)_{3} \mathrm{UCH}_{3}$ was also characterized experimentally with the help of ${ }^{1} \mathrm{H}$ NMR spectroscopy. The NMR spectrum reveals that the proton chemical shifts exhibit strong paramagnetic effects. In reference to tetramethylsilane (TMS), the methyl protons have a paramagnetic NMR $\operatorname{shift}\left(\delta_{\mathrm{pNMR}}^{\mathrm{H}}\right)$ of $-195 \mathrm{ppm}$ at room temperature. [48] The ${ }^{1} \mathrm{H}$ pNMR shifts were calculated fully ab-initio, using restricted active space self-consistent field (RASSCF) wavefunctions as described in Reference 49. The resulting temperature-dependence of $\delta_{\mathrm{pNMR}}^{\mathrm{H}}$ is shown in Figure 0.14-(b). If one assumes that the pNMR ligand shifts, with respect to an analogous diamagnetic system, are principally due to contributions that arise from the low-lying paramagnetic metal-centered states, then the isotropic ligand pNMR shifts can be calculated from the metal-centered low-energy states only, using the Soncini and van den Heuvel $(\mathrm{SvH})$ equation. [51,52] Similar to the van Vleck equation, [50] the SvH equation contains Curie and LR terms to represent the explicitly temperature-dependent and temperature-independent contributions, respectively, to the NMR shifts. As seen in Figure 0.14-(b), the methyl proton pNMR shift in $\left(\mathrm{C}_{5} \mathrm{H}_{5}\right)_{3} \mathrm{UCH}_{3}$ is constant at low $T$. This behavior arises from the LR term of the non-degenerate GS. Despite the fact that the paramagnetic electronic states are not populated at these temperatures, the very strong magnetic coupling between the GS and the lowest ESs renders the methyl proton shifts highly unusual. At higher $T$, the thermal population of the excited states causes $1 / T$-dependent contributions to $\delta_{\mathrm{pNMR}}^{\mathrm{H}}$ from the Curie terms, but they remain relatively small, while the LR contributions to the shift decrease in magnitude with increasing $T$. At room temperature, a methyl proton shift of $-182 \mathrm{ppm}$ is calculated, in quite good agreement with the experiment.

\subsubsection{The $\left[U\left(\operatorname{Tp}^{M e 2}\right)_{2}(\right.$ bipy $\left.)\right]$ I complex.}

$\left[\mathrm{U}\left(\mathrm{Tp}^{\mathrm{Me} 2}\right)_{2}\right.$ (bipy) $] \mathrm{I}$ where $\mathrm{Tp}^{\mathrm{Me} 2}=$ hydrotris(3,5- dimethylpyrazolyl)borate is a complex of U(III) with a $5 f^{3}$ configuration exhibiting Single Molecule Magnet (SMM) behavior [53]. The ground KD presents a roughly axial magnetization in a direction close to the pseudo $C_{2}$ symmetry axis $\left(g_{Z}=3.4, g_{X}=1.3, g_{Y}=0.7\right)$. The first excited KD lies at $138 \mathrm{~cm}^{-1}$ above the ground state.

NOs for the ground KD are depicted in Figure 0.15. All $5 f$ orbitals participate in this state, with an occupation varying from 0.65 to 0.25 . For the sake of comparison, the CASSCF canonical orbitals are represented. The whole splitting of the $5 f$ orbitals is more than $1000 \mathrm{~cm}^{-1}$; the more destabilized orbitals show a larger delocalization in the $\pi$ system of the ligands due to a larger anti-bonding interaction. The trend is the same for the NSOs : the more delocalized orbitals are less populated since higher in energy.

In direction $Z$ with the largest magnetic moment, $g_{Z}=3.4$ with respective orbital and spin contributions of $g_{Z}^{L}=6.23$ and $g_{Z}^{S}=-2.81$. These two contributions have opposite signs, and the orbital one is the largest, as a reminiscence of the free ion where according to the third Hund's rule $J=L-S$ and $L=6$ and $S=3 / 2$. 
Five NSOs have an important $\alpha$ contribution and the $\beta$ contribution almost vanishes. The total spin magnetization for this direction appears quite spherical. Along $X$ and $Y, g_{X}^{L}=1.50, g_{X}^{S}=-0.78, g_{Y}^{L}=2.24$ and $g_{Y}^{S}=-0.96$.. In these directions, the $\beta$ contribution to the spin density is not negligeable. In all directions, the orbital magnetization is mostly a ring around the corresponding axis which spreads on the ligands perpendicular to this axis.

In this case of an unsymmetrical actinide complex with three 'magnetic electrons', wave functions are difficult to analyze since they are strongly multi-determinental, dynamical correlation plays an important role, and the SO coupling mixes many of the SF states. The vizualization tools provide a useful and complementary way in order to gain physical insight and to analyze the magnetic properties of the ground state.

\subsection{Summary}

Magnetic molecules tend to have complicated electronic states. Nonetheless, different sets of orbitals and their populations, generated from the complex manydeterminant wavefunctions, can provide chemically intuitive insight into the chemical bonding and the resulting magnetic behavior. Additionally, the total spin and orbital magnetizations provide information about the magnetic behavior and, for instance, whether the ligands of an open-shell metal center contribute directly to it.

Acknowledgements J.A. acknowledges support of his research on EPR and NMR parameters of actinide complexes, and theoretical method developments, by the U.S. Department of Energy, Office of Basic Energy Sciences, Heavy Element Chemistry program, under grant DE-FG02-09ER16066.

Key words: Electron paramagnetism, electron paramagnetic resonance, spin Hamiltonian, spin orbit coupling, hyperfine coupling, electron $g$-factor, Zeeman interaction, electron spin, nuclear spin, finite nucleus effects

\section{References}

1. G.K.L. Chan, S. Sharma, Annu. Rev. Phys. Chem. 62, 465 (2011)

2. S. Knecht, E.D. Hedegård, S. Keller, A. Kovyrshin, Y. Ma, A. Muolo, C. Stein, M. Reiher, Chimia 70, 244 (2016)

3. S. Knecht, S. Keller, J. Autschbach, M. Reiher, J. Chem. Theory Comput. 12, 5881 (2016)

4. R. Parr, W. Yang, Density-Functional Theory of Atoms and Molecules (Oxford University Press, 1989)

5. L.F. Chibotaru, L. Ungur, J. Chem. Phys. 137, 064112 (2012)

6. P.J. Mohr, B.N. Taylor, D.B. Newell, Rev. Mod. Phys. 84, 1527 (2012)

7. P.O. Löwdin, Phys. Rev. 97, 1475 (1955)

8. B.O. Roos, P.R. Taylor, P.E.M. Siegbahn, Chem. Phys. 48, 157 (1980)

9. P.A. Malmqvist, B.O. Roos, B. Schimmelpfennig, Chem. Phys. Lett. 357, 230 (2002) 
10. F. Aquilante, J. Autschbach, R.K. Carlson, L.F. Chibotaru, M.G. Delcey, L.D. Vico, I. Fdez. Galván, N. Ferré, L.M. Frutos, L. Gagliardi, M. Garavelli, A. Giussani, C.E. Hoyer, G.L. Manni, H. Lischka, D. Ma, P.Å. Malmqvist, T. Müller, A. Nenov, M. Olivucci, T.B. Pedersen, D. Peng, F. Plasser, B. Pritchard, M. Reiher, I. Rivalta, I. Schapiro, J. Segarra-Martí, M. Stenrup, D.G. Truhlar, L. Ungur, A. Valentini, S. Vancoillie, V. Veryazov, V.P. Vysotskiy, O. Weingart, F. Zapata, R. Lindh, J. Comput. Chem. 37, 506 (2016)

11. B.O. Roos, P.R. Taylor, P.E.M. Siegbahn, Chem. Phys. 48, 157 (1980)

12. K. Andersson, P.Å. Malmqvist, B.O. Roos, A.J. Sadlev, K. Wolinski, J. Phys. Chem. 94, 5483 (1990)

13. A. Wolf, M. Reiher, B.A. Hess, J. Chem. Phys. 117(20), 9215 (2002)

14. H. Bolvin, ChemPhysChem 7, 1575 (2006)

15. S. Vancoillie, P.A. Malmqvist, K. Pierloot, ChemPhysChem 8, 1803 (2007)

16. S. Vancoillie, F. Neese, L. Rulisek, K. Pierloot, J. Phys. Chem. A 113, 6149 (2009)

17. K. Ridier, A. Mondal, C. Boileau, O. Cador, B. Gillon, G. Chaboussant, B. Le Guennic, K. Costuas, R. Lescouëzec, Angew. Chem. Int. Ed. 55, 3963 (2016)

18. J. Autschbach, Comments Inorg. Chem. 36, 215 (2016)

19. F. Gendron, B. Pritchard, H. Bolvin, J. Autschbach, Dalton Trans. 44, 19886 (2015)

20. F. Gendron, D. Páez-Hernández, F.P. Notter, B. Pritchard, H. Bolvin, J. Autschbach, Chem. Eur. J. 20, 7994 (2014)

21. W.K. Myers, E.N. Duesler, D.L. Tierney, Inorg. Chem. 47, 6701 (2008)

22. W.K. Myers, C.P. Scholes, D.L. Tierney, J. Am. Chem. Soc. 131, 10421 (2009)

23. A. Abragram, M.H.L. Pryce, Proc. R. Soc. Lond. A 206, 173 (1951)

24. J.P. Jesson, J. Chem. Phys. 45, 1049 (1966)

25. B.R. McGarvey, J. Chem. Phys. 53, 86 (1970)

26. F. Neese, J. Am. Chem. Soc. 128, 10213 (2006)

27. B.R. McGarvey, J. Telser, Inorg. Chem. 51, 6000 (2012)

28. H. Andres, E.L. Bominaar, J.M. Smith, N.A. Eckert, P.L. Holland, E. Münck, J. Am. Chem. Soc. 124, 3012 (2002)

29. H. Bolvin, J. Autschbach, in Handbook of Relativistic Quantum Chemistry, ed. by W. Liu (Springer, Berlin, 2017), pp. 725-763

30. G. Rogez, J.N. Rebilly, A.L. Barra, L. Sorace, G. Blondin, N. Kirchner, M. Duran, J. van Slageren, S. Parsons, L. Ricard, A. Marvilliers, T. Mallah, Angew. Chem. 44, 1876 (2005)

31. G. Charron, E. Malkin, G. Rogez, L.J. Batchelor, S. Mazerat, R. Guillot, N. Guihèry, A.L. Barra, T. Mallah, , H. Bolvin, Chem. Eur. J. 22, 1 (2017)

32. M.D. Walter, C.H. Booth, W.W. Lukens, R.A. Andersen, Organomet. 28(3), 698 (2009)

33. J.D. Rinehart, J.R. Long, Chem. Sci. 2, 2078 (2011)

34. K.R. Meihaus, J.R. Long, J. Am. Chem. Soc. 135, 17952 (2013)

35. Y.C. Chen, J.L. Liu, W. Wernsdorfer, D. Liu, L.F. Chibotaru, X.M. Chen, M.L. Tong, Angew. Chem. Int. Ed. 56, 4996 (2017)

36. T. Kajwara, Angew. Chem. Int. Ed. 56, 10.1002/anie.201703022 (2017)

37. J. Dognon, Pyykkö, Angew. Chem. Int. Ed. 56, 10132 (2017)

38. J.U. Reveles, P.A. Clayborne, A.C. Reber, S.N. Khanna, K. Pradhan, P. Sen, M.R. Pederson, Nature Chem. 1, 310 (2009)

39. P. Pyykkö, C. Clavaguéra, J. Dognon, in Computational Methods in Lanthanide and Actinide Chemistry, ed. by M. Dolg (Wiley-VCH, 2015)

40. X. Zhang, Y. Wang, H. Wang, A. Lim, G. Gantefoer, K.H. Bowen, J.U. Reveles, S.N. Khanna, J. Am. Chem. Soc. 135, 4856 (2013)

41. C.A.J. Hutchison, B. Weinstock, J. Chem. Phys. 32, 56 (1960)

42. C.A.J. Hutchison, T. Tsang, B. Weinstock, J. Chem. Phys. 37, 555 (1962)

43. F.P. Notter, H. Bolvin, J. Chem. Phys. 130(18), 184310 (2009)

44. F. Gendron, B. Le Guennic, J. Autschbach, Inorg. Chem. 53, 13174 (2014)

45. N.A. Siladke, K.R. Meihaus, J.W. Ziller, M. Fang, F. Furche, J.R. Long, W.J. Evans, J. Am. Chem. Soc. 134, 1243 (2012)

46. O. Kahn, Molecular magnetism (VCH, New York, 1993) 
47. D.R. Kindra, W.J. Evans, Chem. Rev. 114, 8865 (2014)

48. T. Marks, A.M. Seyam, J. Am. Chem. Soc. 94(18), 6545 (1972)

49. F. Gendron, J. Autschbach, J. Chem. Theory Comput. 12, 5309 (2016)

50. J. Autschbach, in Annual Reports in Computational Chemistry, vol. 11, ed. by D.A. Dixon (Elsevier, Amsterdam, 2015), pp. 3-36

51. W. Van den Heuvel, A. Soncini, J. Chem. Phys. 138, 054113 (2013)

52. A. Soncini, W. Van den Heuvel, J. Chem. Phys. 138(2), 021103 (2013)

53. J.T. Coutinho, M.A. Antunes, L.C.J. Pereira, H. Bolvin, J. Marçaloa, M. Mazzanti, M. Almeida, Dalton Trans 41, 13568 (2012) 


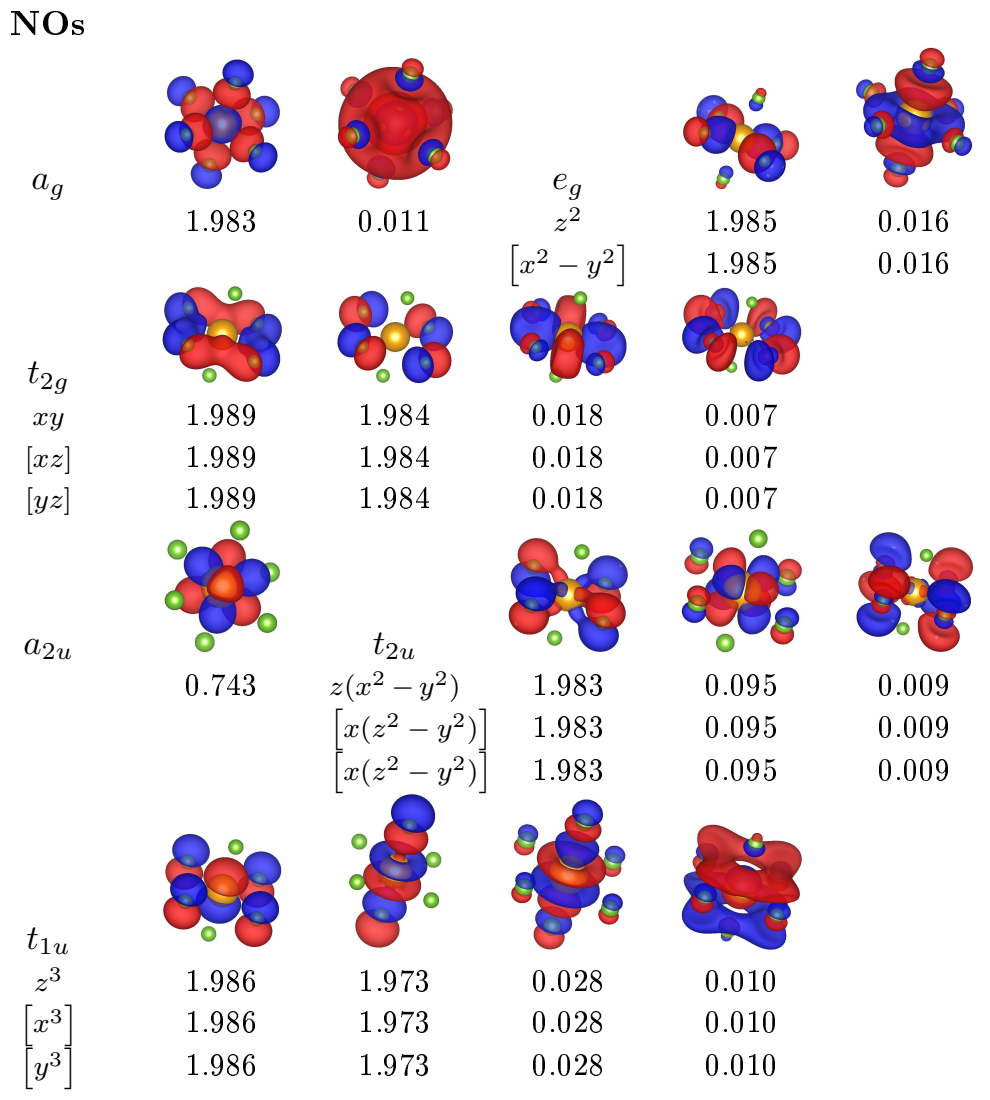

NSOs

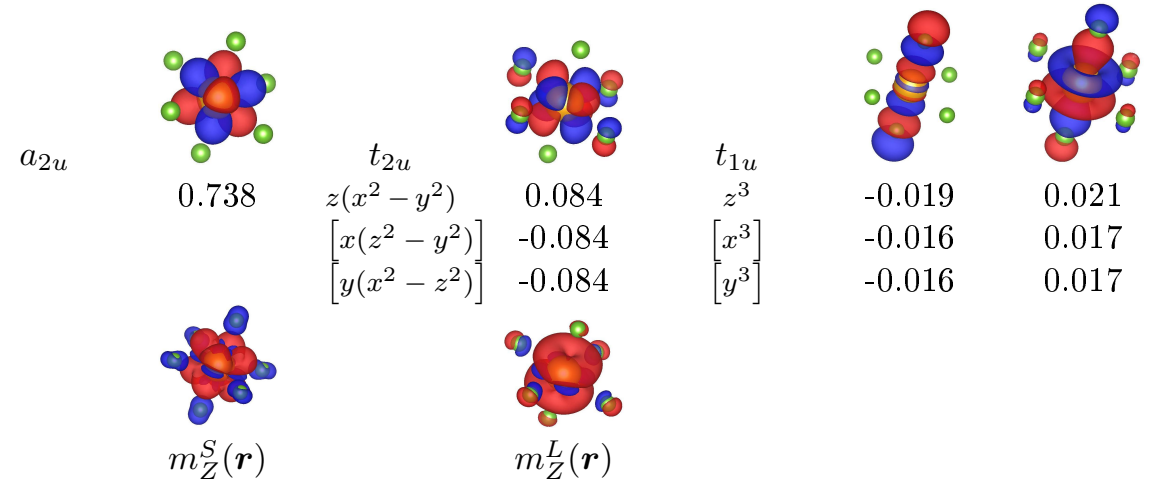

Fig. 0.12 Isosurface of selected NOs and NSOs along the $z$ axis and corresponding populations. One orbital is represented per degenerate irrep, the components in brackets may be deduced by symmetry. The NSOs along $X$ and $Y$ are obtained by circular permutation. Isovalues: \pm 0.04 au for orbitals, \pm 0.0008 au for magnetization. 


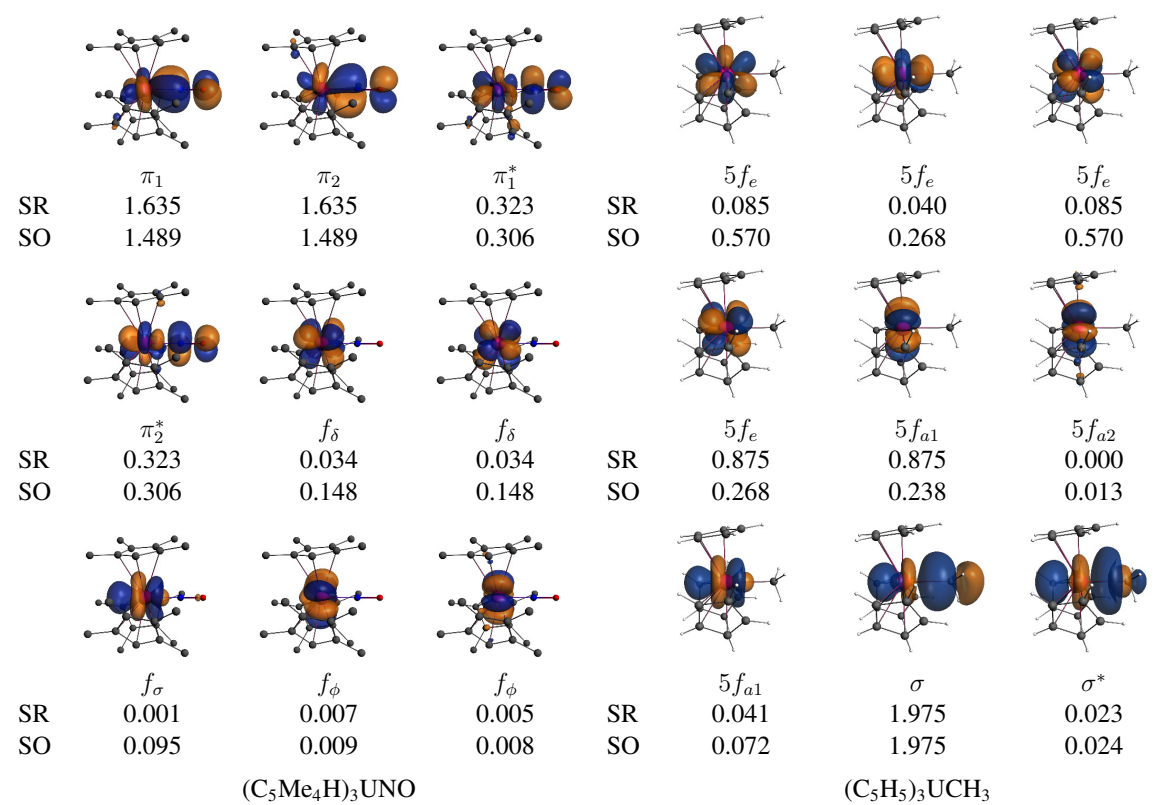

Fig. 0.13 Selected SR NOs and occupations for the SR and SO GS of $\left(\mathrm{C}_{5} \mathrm{Me}_{4} \mathrm{H}\right)_{3} \mathrm{UNO}$ (left) and $\left(\mathrm{C}_{5} \mathrm{H}_{5}\right)_{3} \mathrm{UCH}_{3}$ (right). CAS $(4,9) \mathrm{SCF}$ Results. Isosurfaces $( \pm 0.03 \mathrm{au})$. For a sake of clarity, hydrogen atoms have been removed in $\left(\mathrm{C}_{5} \mathrm{Me}_{4} \mathrm{H}\right)_{3} \mathrm{UNO}$. The SO NOs appear very similar to the SR NOs; the main difference between the SR and SO calculations is seen in the occupation numbers.

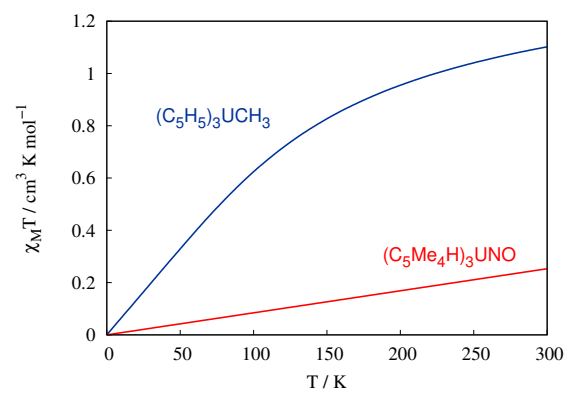

(a)

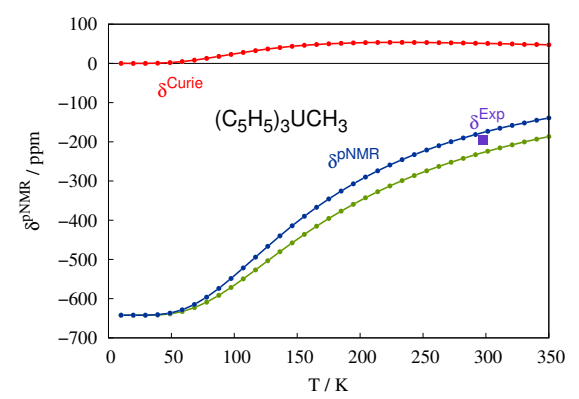

(b)

Fig. 0.14 (a) Calculated magnetic susceptibility times the temperature, $\chi T\left(\mathrm{~cm}^{3} \mathrm{~K} \mathrm{~mol}^{-1}\right)$, as a function of $T(\mathrm{~K})$ for $\left(\mathrm{C}_{5} \mathrm{Me}_{4} \mathrm{H}\right)_{3} \mathrm{UNO}$ and $\left(\mathrm{C}_{5} \mathrm{H}_{5}\right)_{3} \mathrm{UCH}_{3}$. (b) Calculated temperature-dependence of the ${ }^{1} \mathrm{H}$ pNMR shift $\delta^{\mathrm{pNMR}}(\mathrm{ppm})$ for $\left(\mathrm{C}_{5} \mathrm{H}_{5}\right)_{3} \mathrm{UCH}_{3}$ and individual LR and Curie contributions. The experimental shift at room temperature is also indicated. 


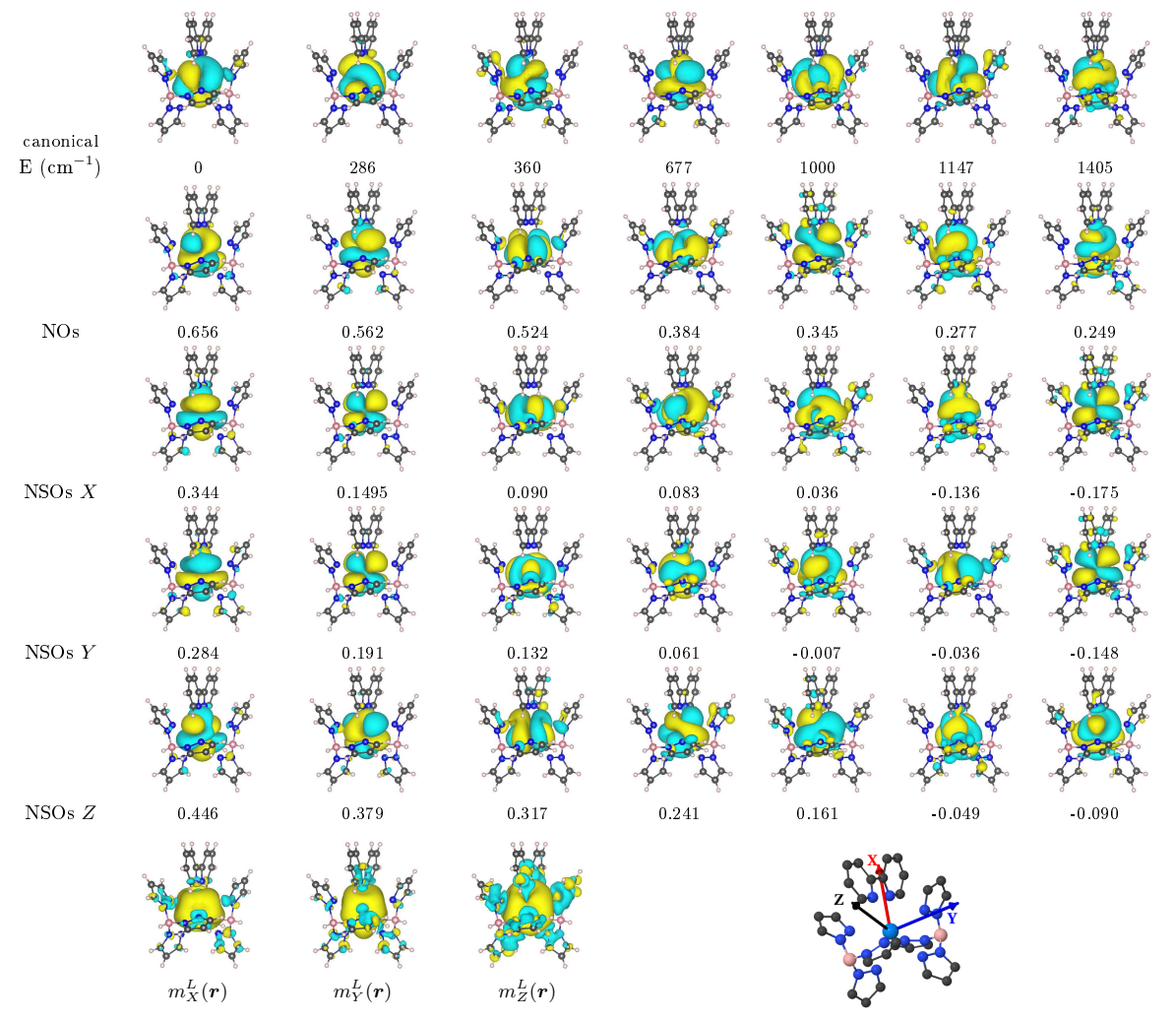

Fig. 0.15 For $\left[\mathrm{U}\left(\mathrm{Tp}^{\mathrm{Me} 2}\right)_{2}\right.$ (bipy)]I ; Top row: Isosurface and energy $\left(\mathrm{cm}^{-1}\right)$ of the $5 f$ canonical orbitals. Second row: Isosurface and occupation number of selected NOs. Third to fifth row: Isosurface and spin population of NSOs for the three magnetic axes. Bottom row: Isosurface of the orbital magnetization $m_{U}^{L}(\boldsymbol{r})$ ) for the three magnetic axes and representation of the magnetic axes. NOs and NSOs are calculated for the component of the ground KD with $\left\langle S_{U}\right\rangle>0$. Isovalues: \pm 0.04 au for orbitals, \pm 0.0001 au for magnetization. 